\title{
Magnetorheological effect in the magnetic field oriented along the vorticity
}

\author{
P. Kuzhir ${ }^{1}$ C. Magnet ${ }^{1}$, L. Rodríguez-Arco ${ }^{2}$, M.T. López-López ${ }^{2}$, H. Fezai ${ }^{1}$, A.Meunier ${ }^{1}$, \\ A.Zubarev ${ }^{3}$, and G. Bossis ${ }^{1}$ \\ ${ }^{1}$ University of Nice-Sophia Antipolis, CNRS UMR7336, Laboratory of Condensed Matter Physics, 28, avenue \\ Joseph Vallot, 06100 Nice, France \\ ${ }^{2}$ Department of Applied Physics, University of Granada, Campus de Fuentenueva, 18071 Granada, Spain \\ ${ }^{3}$ Department of Mathematical Physics, Ural Federal University, 51, Prospekt Lenina 620083, Ekaterinburg, \\ Russia
}

\section{Synopsis}

In this work, we have studied the magnetorheological (MR) fluid rheology in the magnetic field parallel to the fluid vorticity. Experimentally, the MR fluid flow was realized in the Couette coaxial cylinder geometry with the magnetic field parallel to the symmetry axis. The rheological measurements were compared to those obtained in the cone-plate geometry with the magnetic field perpendicular to the lower rheometer plate. Experiments revealed a quasi-Bingham behavior in both geometries with the stress level being just a few dozens of percent smaller in the Couette cylindrical geometry at the same internal magnetic field. The unexpectedly high MR response in the magnetic field parallel to the fluid vorticity is explained by stochastic fluctuations of positions and orientations of the particle aggregates. These fluctuations are induced by magnetic interactions between them. Once misaligned from the vorticity direction, the aggregates generate a high stress independent of the shear rate, and thus assimilated to the suspension apparent (dynamic) yield stress. Quantitatively, the fluctuations of the aggregate orientation are modeled as a rotary diffusion process with a diffusion constant proportional to the mean square interaction torque. The model gives a satisfactory agreement with the experimental field dependency of the apparent yield stress and confirms the nearly quadratic concentration dependency $\sigma_{Y} \propto \Phi^{2.2}$, revealed in experiments. The practical interest of this study lies in the development of MR smart devices with the magnetic field non-perpendicular to the channel walls.

\section{Introduction}

Magnetorheological (MR) fluids are suspensions of micron-sized magnetizable particles dispersed in a liquid carrier. In the absence of an applied magnetic field, they almost behave as classic hard sphere suspensions. When an external magnetic field is applied, the particles acquire a magnetic moment, attract each other and form structures aligned with the field direction. If the applied magnetic field is perpendicular to the walls of the flow channel, the structures oppose a high hydraulic resistance to the flow, which results in a several orders of magnitude increase of the MR fluid viscosity. Furthermore, a significant threshold shear stress - the yield stress - is required to onset the flow. Such a field-induced yielding (Bingham) behavior is referred to as the MR effect [Shulman and Kordonski (1982), Ginder et al. (1998), Bossis et al. (2002b), de Vicente et al. (2011)]. The MR effect is applied in active 
vibro-protection and lubrication systems as well as in precise polishing [Carlson et al. (1996), Kordonski and Jacobs (1996), Urreta et al. (2010)].

Due to the field-induced anisotropy, the MR effect depends not only on the magnetic field strength but also on its orientation. The MR fluid rheological behavior has been extensively studied for the case of magnetic fields perpendicular to both the fluid velocity and the vorticity. Experimentally, this configuration has been realized in different ways: (a) using cone-plate or plate-plate rotational rheometry with an axial magnetic field [cf. reviews by Shulman and Kordonski (1982), Ginder et al. (1998)]; (b) in Couette concentric cylinder or cylindrical bob and cup rheometers with a radial magnetic field [Shulman and Kordonski (1982), Ginder et al. (1996), Laun et al. (1996), Vekas et al. (2001), Genç and Phulé (2002), Ulicny et al. (2005), Kordonski and Gorodkin (2009)]; (c) in pressure driven flows through plane, cylindrical or annular channels with a magnetic field applied normally to the channel walls [Shulman and Kordonski (1982), Gavin (2001), Kuzhir et al. (2003), Wang and Gordaninejad (2006), Ocalan and McKinley (2012)]. Theoretically, the MR effect in this configuration is well understood in terms of both the loss of contact between the rheometer walls and the percolating particle structures (responsible for the static yield stress), and the viscous dissipation on the particle aggregates whose size decreases with the shear rate (responsible for the dynamic yield stress). See the review by Bossis et al. (2002b) and the references therein for further information.

The studies in other magnetic field orientations are less documented. As far as we know, pioneering works on the effect of the magnetic field orientation on the MR fluid rheology belong to the group of Profs. Shulman and Kordonski. They have shown a somewhat stronger MR response in the pipe flow under a transverse applied field as compared to the longitudinal field [Shulman and Kordonski (1982)]. They have also realized a flow through a slit channel subjected to a magnetic field either normal to the channel walls or parallel to the fluid vorticity, reporting a somewhat weaker MR response for the second configuration [Kordonski et al. (1989)]. Later on, Bossis et al. (2002a) have carried out comparative experiments for: (a) the plate-plate geometry with the magnetic field perpendicular to the walls; and (b) the Couette concentric cylinder geometry with an axially applied magnetic field, i.e. the field aligned with the fluid vorticity. The MR fluid has shown nearly the same MR response in both geometries at the same internal magnetic field, taking into account the quite strong demagnetizing field in the parallel plate geometry. Takimoto et al. (1999) have reported a 2.5 times decrease of the field-induced yield stress in a plane channel as the magnetic field orientation is progressively varied from the transverse to the longitudinal one. Such an angular dependency of the MR effect has been later reproduced for pressure-driven flows by Kuzhir et al. (2003) and understood in terms of the progressive decrease of the angle between the particle aggregates and the flow as the angle between the applied magnetic field and the flow varies from $90^{\circ}$ to $0^{\circ}$. However, none of the above cited works give a clear explanation to the yield stress existence in magnetic fields parallel to either the fluid velocity or to the fluid vorticity. 
At first glance, the particle structures are expected to be aligned with the direction of the field when this latter is oriented along either the fluid velocity or the fluid vorticity. This would result in a relatively low viscous dissipation and low stress levels. Let us examine in more details the configuration with the magnetic field aligned with the vorticity - the case of the present study. If we consider a dilute regime and assume that the particle aggregates do not interact with each other, we may describe their angular motion by Jeffery equations with an external torque included. Chaffey and Mason (1964) and Almog and Frankel (1995) have carried out these calculations for non-Brownian rod-like particles. They have shown that, whatever the field strength is, initially misaligned particles perform precessional motion around the vorticity axis and their trajectories converge to the steady-state orientation aligned with the vorticity (and field) direction. Thus, in the steady-state regime, the rheology of the suspension of very long and fully aligned aggregates is expected to be similar to that of a suspension of infinite cylinders aligned with the vorticity. The viscosity of such a suspension depends on the particle concentration [Christensen (1991), Christensen (1993)] but should be independent of the magnetic field intensity as far as the rotation of aggregates around their axis is not affected by the field and fully determines the suspension stress. Thus, no yield stress is expected. Of course, here we consider magnetic fields strong enough to induce particle aggregation (typically $H>1 \mathrm{kA} / \mathrm{m}$ ).

In a typical MR fluid with a particle volume fraction of about $10 \%$, interactions between particle aggregates become important and induce periodic misalignments of aggregates with respect to the vorticity direction. Misaligned aggregates could generate substantial viscous stresses depending on the aggregate orientation distribution and length, which depend, in their turn, on the interplay between the magnetic and hydrodynamic interactions. Recently, Kuzhir et al. (2011) have theoretically studied the MR fluid flow in the longitudinal field and shown that stochastic aggregate misalignments from the flow direction (caused by magnetic interactions between aggregates) could result in a Bingham behavior with an apparent (dynamic) yield stress comparable to that measured in the transverse field. We expect therefore that the same physics may govern the MR fluid rheology in the magnetic field parallel to the fluid vorticity. Recall that the considered mechanism requires relatively strong interactions between aggregates. Otherwise, the effect can be the opposite, i.e., a decrease of the effective viscosity with the increasing field, as it has been observed in suspensions of weakly magnetizable red blood cells [Tao and Huang (2011)].

In the present paper, we perform both theoretical and experimental studies on the MR fluid flow in the magnetic field parallel to the fluid vorticity. In the experiments, we use a concentric cylinder rotational geometry with an axially applied magnetic field, and make the measurements in a wide range of applied magnetic fields and MR fluid concentrations. A special attention is paid to the comparison of the MR effect for the magnetic field parallel to the fluid vorticity and parallel to the velocity gradient, the latter configuration being realized with the help of a cone-plate geometry. In our theory, we model the stochastic misalignments of the particle aggregates as a rotary diffusion process with a diffusion constant defined by the strength of the magnetic interactions between aggregates. Our main goal is to demonstrate 
that such a field-induced rotary diffusion process may be at least one of the possible mechanisms explaining the MR effect in the magnetic field aligned with the fluid vorticity.

Apart from the fundamental interest, this study is motivated by the development of active hydrodynamic/hydrostatic bearings of machine tools with the magnetic field applied coaxially with the rotating shaft. In particular, this configuration corresponds to bearings equipped with a non-magnetic shaft [Urreta et al. (2010)]. The use of non-magnetic shafts instead of magnetic ones allows avoiding the problem of magnetic attraction between the shaft and the pole pieces of the electromagnet, which hinders an effective real-time control over the bearing performances.

The present paper is organized as follows. In Section II, we present the experimental apparatus and experimental protocols. In Section III, we overview the experimental results obtained both in the Couette cylindrical and the cone-plate geometries. The theoretical model for the MR effect in the magnetic field aligned with the vorticity is described and compared with experiments in Section IV. Conclusions and perspectives are outlined in Section V.

\section{Experimental procedure}

The magnetorheological fluids of particle volume fractions ranging from 10 to $36 \%$ were prepared by dispersing carbonyl iron (BASF) microparticles (of a mean diameter of 2 $\mu \mathrm{m}$ and magnetization saturation, $M_{S}=1.36 \cdot 10^{6} \mathrm{~A} / \mathrm{m}$ ) in a silicon oil (Rhodorsil ${ }^{\circledR} 47 \mathrm{~V} 500$; VWR International, dynamic viscosity at $25^{\circ} \mathrm{C}$ is $\eta_{0}=0.48 \mathrm{~Pa} \cdot \mathrm{s}$ ) under vigorous stirring. Subsequently, the MR fluids were stabilized against aggregation due to colloidal interactions by adding an appropriate amount of aluminum stearate (Sigma Aldrich), as described in details in López-López et al. (2008).

The rheological measurements were performed with the help of the controlled-stress rotational rheometer Thermo Haake RS 150 using a standard titanium-made cylindrical Couette geometry (bob and cup geometry Z20 DIN/ISO 3219) shown schematically in Fig. 1. The internal cylinder had a diameter of $D=20 \mathrm{~mm}$ and height $h=30 \mathrm{~mm}$; the radial distance between both cylinders (called hereinafter the gap) was equal to $g=0.82 \mathrm{~mm}$. The bottom cone had an apex angle $2 \varphi=118^{\circ}$ and the distance between the cone edge and the bottom of the cup was adjusted to $h_{1}=1 \mathrm{~mm}$. The amount of MR sample poured into the cup was chosen in such a way to keep the same distance (equal to $h_{2}=15 \mathrm{~mm}$ ) between its free surface and the upper bob surface for all experiments. A uniform external magnetic field was applied co-axially with the measuring geometry, and thus, in the direction parallel to the MR fluid vorticity. This field was generated by a coil ( $80 \mathrm{~mm}$ in internal diameter and 130 in height) placed co-axially with the measuring geometry. The magnetic field $H_{0}$ was measured in the center of the empty coil with the help of a Caylar GMH-103 gaussmeter as a function of the electric current $I$ applied to the coil, and the following linear relationship was established: $H_{0}=6.11 \cdot I$, where $I$ is expressed in $\mathrm{A}$ and $H_{0}$ in $\mathrm{kA} / \mathrm{m}$. 


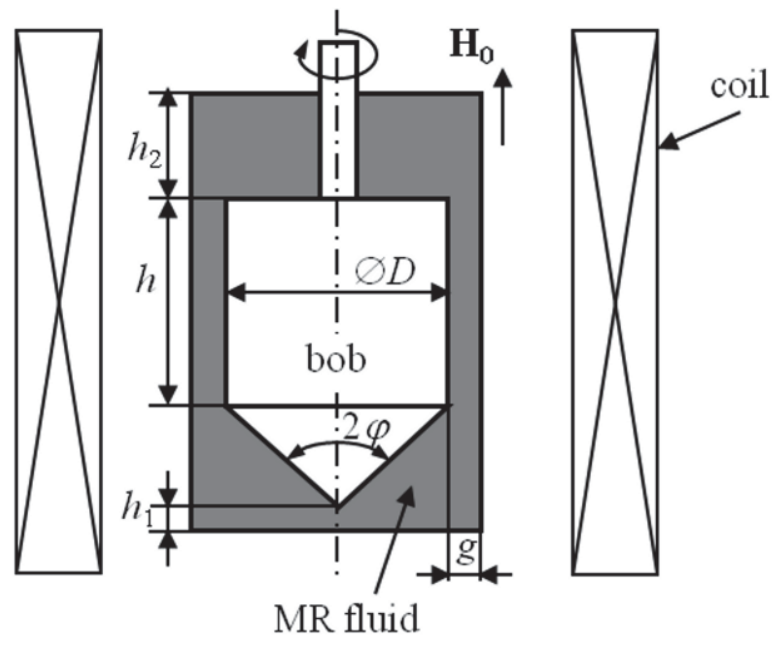

Fig.1. Cylindrical Couette (bob and cup) geometry with the magnetic field applied parallel to the rheometer axis

The measuring protocol can be summarized as follows. Before the measurements the MR fluids were degasified for 15 minutes and placed immediately after that inside the rheometer. Firstly, the MR fluid was pre-sheared in the absence of the magnetic field for 5 minutes at a shear rate equal to $100 \mathrm{~s}^{-1}$. Then, a magnetic field of an intensity of about $6 \mathrm{kA} / \mathrm{m}$ was applied and the pre-shear stage continued for another 5 minutes. After the pre-shear stage, the magnetic field intensity was adjusted to a desired value (ranging from 6 to 30.6 $\mathrm{kA} / \mathrm{m}$ ), the suspension was left at rest for 3 minutes, and then, a stress ramp was applied to the MR sample with duration of each step equal to $30 \mathrm{~s}$. The shear rate was measured for each step of the stress ramp and the dependency of the shear stress on the shear rate - the flow curve - was obtained. Once the stress ramp ended, the magnetic field was again readjusted to the value of $6 \mathrm{kA} / \mathrm{m}$, and the pre-shear stage was repeated before a new stress ramp performed at a new value of the applied field. Note that the magnetic field (of an intensity of at least 6 $\mathrm{kA} / \mathrm{m}$ ) was maintained from the beginning to the end of the measurements in order to reduce particle settling because of gravity. We have checked that the MR fluid viscosity at the end of the measurements was, in the worst case, $10 \%$ lower than that at the beginning.

It is important to note that the measured flow curves correspond to the shear flow in the whole MR sample, covering not only the measuring gap between cylindrical parts of the geometry but also the regions below and above the measuring gap. Thus, the values of the imposed stress need to be corrected by subtracting eventual contributions of the bottom and upper parts of the sample. The contribution of the upper part is deduced by comparing the measured friction torques (exerted by the MR fluid on the rotating bob) for different heights $h_{2}$ above the upper bob surface [cf. Fig.1]. This contribution appears to be lower than the instrumental error of the rheometer of $1 \%$. The contribution of the bottom part is inspected by measurements of the friction torque for the MR samples filling the cup up to the level, at which the bottom cone is fully immerged into the MR fluid but the measuring gap remains unfilled. This torque appears to be about $2 \%$ of the total torque corresponding to the whole bob immersed into the sample. To correct this systematic error, we subtracted the friction torque $T_{\text {cone }}$ measured for the immersed cone from the torque $T_{b o b}$ measured for the fully 
immersed bob. The shear stress in the gap between rotating cylinders is therefore given by: $\sigma=\left(T_{\text {bob }}-T_{\text {cone }}\right) /\left(2 \pi R^{2} L\right)$, with $R=10 \mathrm{~mm}$ and $L=30 \mathrm{~mm}$ being, respectively, the radius and the length of the cylindrical part of the rotating bob.

When the material undergoes a yielding behavior, the friction torque exerted on a considered part of the bob is expected to be proportional to the product of the yield stress, $\sigma_{Y}$, and the geometry radius, $r$, and the lateral surface, $S$, of the respective bob part. The product $S \cdot r$ appears to be much smaller for the bottom and the upper parts of the bob as compared to the central cylindrical part corresponding to the measuring gap. Furthermore, analysis shows that the average magnetic field in the upper and the bottom parts is a few times lower than in the central part (cf. Appendix B); this could result in lower values of the yield stress in these parts as compared to the central part. Thus, combination of these both effects could explain the negligible measured contributions of the bottom and the upper parts of the sample to the overall friction torque.

In order to compare the rheological results obtained for different magnetic field orientations, we also conducted the rheological measurements in a cone-plate geometry (made of titanium, diameter $35 \mathrm{~mm}$ and apex angle $2^{\circ}$ ) in the presence of a uniform magnetic field aligned with the rheometer axis and generated by the same coil placed co-axially with the measuring geometry. We used a measuring protocol similar to the one used for the cylindrical Couette geometry without performing any rheometric corrections.

\section{Experimental results}

\section{A. Analysis of the internal field}

The main goal of this Section is to confirm the effect of the magnetic field orientation (either along the vorticity or along the velocity gradient) on the MR response of the MR suspensions within a wide range of experimental parameters, such as the shear rate, the magnetic field intensity and the suspension concentration. For this purpose, we will compare rheological results obtained both in Couette and cone-plate geometries, in which the field is aligned along the vorticity and along the velocity gradient, respectively. Comparison at the same external magnetic field does not allow a correct analysis of the intrinsic MR properties of the suspension because these properties depend principally on the internal magnetic field, $H$, inside the MR sample rather than on the external one $H_{0}$. Therefore, we need to determine the distribution of the internal magnetic field as a function of the shape of the MR sample and of the applied homogeneous field, $H_{0}$. The internal field $H$ depends, among other things, on the suspension microstructure, which, in its turn, is strongly affected by the shear rate. Direct measurements of the magnetic field distribution inside narrow gaps of the sheared sample are subjected to various technical problems. Alternatively, the field distribution can be obtained by finite element calculations, which require knowledge of the magnetic permeability, $\mu$, of the suspension as a function of the field and the shear rate. Experimental determination of $\mu$ in sheared suspensions poses problems similar to those related to measurements of $H$. Therefore, 
we shall proceed to theoretical estimations of the suspension permeability using two different approaches.

In our experiments, the yield stress was obtained by a linear fit of the flow curves at relatively high shear rates. Therefore, both estimations were done for the high-shear regime, i.e. at shear rates $\dot{\gamma}>100 \mathrm{~s}^{-1}$. The first estimation is based on the assumption of completely unstructured suspension at high shear rates. This situation corresponds to an isotropic state with particle structures destroyed by shear, as opposed to the low-shear regime with strongly aligned structures. De Vicente et al. (2002) have reported values of the magnetic permeability of the unstructured MR suspension at different particle volume fractions, $\Phi$ [see Fig. 5 of that paper]; for example, $\mu \approx 2.6$ at $\Phi=0.3$. The second estimation is based on the assumption of elongated aggregates. In the case of the Couette cylindrical geometry, the aggregates are supposed to be aligned with both the external field and the fluid vorticity (in reality, the aggregate orientation may fluctuate around the vorticity axis - see Sec. IV - but we neglect these fluctuations for estimations of $\mu$ ). At such conditions, the suspension properties possess a strong anisotropy and the axial, $\mu_{\mathrm{zz}}$, and radial, $\mu_{r r}$, components of the permeability tensor are found using the Maxwell-Garnett mean field theory [Berthier (1993)], as described in details in Appendix A. In the case of the cone-plate geometry, we adopt the model of cylindrical particle aggregates tilted by the shear flow at some angle $\phi$ with respect to the applied field. This model has been developed by Gómez-Ramírez et al. (2011) [Eqs. (25), (26) of that paper] and the basic equations are presented in Appendix A. The model provides a value of the tilt angle as a function of the applied field [Eq. A.3], but independent of the shear rate in the high-shear regime. Using this estimated orientation of the aggregates, we calculate the components of the magnetic permeability tensor [Eqs. A.4-A.5]. This second estimation provides the following mean values (averaged over the range of the external field $0<\mathrm{H}_{0}<30.6$ $\mathrm{kA} / \mathrm{m}$ ) of the magnetic permeability components at $\Phi=0.3: \mu_{\mathrm{zz}} \approx 5.8$ and $\mu_{\mathrm{rr}} \approx 2.7$ for the Couette geometry, and $\mu_{\mathrm{zz}} \approx 2.8$ and $\mu_{\mathrm{rr}} \approx 2.7$ for the cone-plate geometry. Analysis shows that the magnetic field distribution in cone-plate geometry is much more sensitive to the magnetic permeability values than the field in the measuring gap of the Couette geometry. Fortunately, both estimations give nearly the same value of the magnetic permeability for the cone-plate geometry. Therefore, for this geometry, we took an average value of the magnetic permeability, $\mu_{z z}=\mu_{r r}=2.7 \pm 0.1$ for estimations of the internal magnetic field.

Once the magnetic permeability is estimated, we proceeded to calculation of the magnetic field distribution inside the MR samples in both considered geometries. Details of the simulations are given in the Appendix B along with Figures B1 and B2 showing the magnetic field distribution. Figure B2 shows that the internal field remains nearly homogeneous in the whole sample filling the cone-plate geometry except near the sample meniscus. The mean value of the internal field is only $5 \%$ higher than the value $H_{0} / \mu_{z z}$ estimated under the assumption of a plain infinitely thin layer of MR fluid [Fig. B2b]. Figure B1 shows that the magnetic field remains nearly homogenous within the measuring gap between two cylinders in the cylindrical Couette geometry. The mean value of the internal 
field in the measuring gap appears to be only $4 \%$ higher than the value $H_{0}$ of the external field measured in the center of the empty coil [Fig. B1b].

Despite this relative homogeneity inside the measuring gap, the magnetic field outside the gap (in the space between the bottom cone and the cup as well as above the bob), is a few times lower than the one inside the gap. This difference appears purely because of the specific shape of the MR fluid sample filling the bob and cup geometry (demagnetizing effect) and it is not related to magnetic parts of the geometry (made of titanium). Such non-uniformity of the magnetic field outside the measuring gap could induce migration of the magnetic particles toward the region of the highest field, i.e. inside the measuring gap, which would result in a considerable increase of the suspension viscosity with time. To check this point, we performed a kind of creep test for which a constant shear stress was applied to the MR sample and the magnetic field was abruptly switched on. The shear viscosity was measured as a function of time during a period of $1 \mathrm{~h}$. The results of this test (not shown here for brevity) revealed a small but gradual viscosity decrease with time at long time scale, totally inconsistent with the particle crowding effect. The insignificance of particle migration is also consistent with an estimation of the characteristic time $t$ of particle migration from the upper or the lower parts of the Couette geometry to the measuring gap. A simple balance of the magnetic force and the hydrodynamic drag acting on the particles gives values of $t$ of the order of $t=\left(3 \eta_{0} X^{2}\right) /\left(2 \beta \mu_{0} H^{2} a^{2}\right) \sim 10^{4}-10^{5} \mathrm{~s}-$ the period strongly exceeding typical experimental times, where $\eta_{0}=0.5 \mathrm{~Pa} \cdot \mathrm{s}$ is the suspending liquid viscosity, $X \sim 10 \mathrm{~mm}$ is a characteristic scale of the field gradient, $a \sim 1 \mu \mathrm{m}$ is the particle radius, $\beta \sim 1$ is the particle magnetic contrast factor, and $\mu_{0}=4 \pi \cdot 10^{-7} \mathrm{H} / \mathrm{m}$ is the magnetic permeability of vacuum.

\section{B. MR response in Couette and cone-plate geometries}

Let us first examine the MR response of the MR suspension in both geometries at the same external magnetic field $H_{0}$. The flow curves obtained in these geometries are shown in Fig.2a for the MR fluid of particle volume fraction $\Phi=0.3$ and at an external magnetic field $H_{0}=18.3 \mathrm{kA} / \mathrm{m}$. Recall that in the first case, the magnetic field is oriented along the vorticity, while in the second one, along the velocity gradient. As it is seen in this figure, the stress level in the Couette geometry is higher than that in the cone-plate geometry at the same external magnetic field. This difference can be attributed to two different effects: (a) the demagnetizing effect of the MR sample, which leads to a substantial difference in internal magnetic fields in both geometries subjected to the same external field; (b) MR fluid anisotropy, i.e. dependence of the suspension properties on the field direction caused by the anisotropy of the MR microstructure. As mentioned in Sec. III-A, the comparison of the MR response at the same external magnetic field does not allow separating these two effects. On the contrary, comparison at the same internal field allows us to eliminate (within the errors related to the theoretical determination of this field) the first effect and to analyze the effect of the field orientation. The flow curves measured for both geometries at the same internal magnetic field, $H \approx 12 \mathrm{kA} / \mathrm{m}$ are shown in Fig. $2 \mathrm{~b}$ for the same particle volume fraction $\Phi=0.3$. As a characteristic value of $H$ in both geometries, we use the average value of the magnetic 
field in the measuring gap of the considered geometry, calculated in Appendix B and shown in Figs. B1b and B2b. Analysis of Figs. 2a and 2b shows that, if the stress level is typically higher in Couette geometry at the same external field (Fig. 2a), the tendency reverses if the comparison is made at the same internal field (Fig. 2b): the stress level in the Couette geometry appears to be lower than that in the cone-plate one. This difference could be explained in terms of the orientation of the suspension microstructure with respect to the flow. The degree of misalignment between the flow and the aggregates in the cone-plate geometry is expected to be higher than the deviation of the aggregate orientation from the vorticity axis in the Couette geometry. Stronger misalignment generates higher viscous dissipation and should result in higher stress level in the cone-plate geometry with the field perpendicular to the rheometer walls.
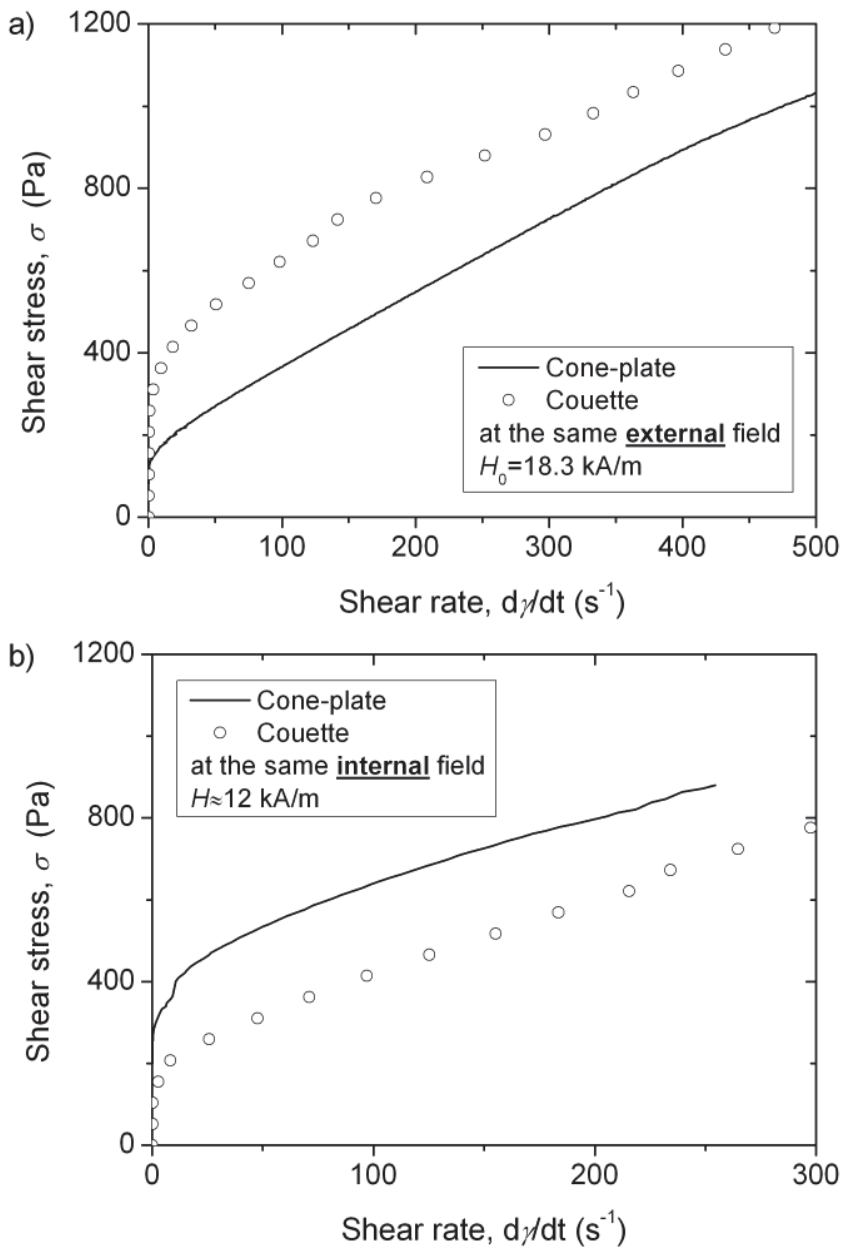

Fig.2. Comparison between the MR fluid flow curves measured in the cone-plate and the cylindrical Couette geometries at the same external magnetic field $H_{0}=18.3 \mathrm{kA} / \mathrm{m}(\mathrm{a})$, and at the same internal magnetic field $\mathrm{H} \approx 12$ $\mathrm{kA} / \mathrm{m}$ (b). For both figures, the particle volume fraction is $\Phi=0.3$

We also remark that the initial part of the flow curve in the cylindrical Couette geometry has a more rounded shape than that in the cone-plate geometry. Such an initial rounded shape of the flow curves has already been observed in magnetic fiber suspensions in the plate-plate geometry and has been attributed to gap-spanning aggregates whose length is 
bounded by the rheometer gap [Gómez-Ramírez et al. (2011)]. The flow curves observed in the present study are likely governed by the same physics (i.e. interactions between the aggregates and the walls); however, at this stage, we are unable to answer why the flow curve in the Couette geometry is smoother than the one in the cone-plate geometry. Finally note that the flow curves shown in Figs. 2a-b exhibit a quasi-linear behavior at relatively high shear rates, $\dot{\gamma}>100 \mathrm{~s}^{-1}$. The high-shear parts of the flow curves were fitted by a linear function, $\sigma=\sigma_{Y}+\eta \dot{\gamma}$, extrapolated to lower shear rates. The intercept, $\sigma_{Y}$, of the linear fit to zero shear rate is defined as the apparent yield stress (often used in magnetorheological literature and sometimes called the dynamic yield stress). The value $\sigma_{Y}$ should not be confounded with the real yield stress (usually called the static yield stress) corresponding to the failure of the percolated MR structures and to the onset of the flow.

This apparent yield stress is considered to be the most important characteristic of the MR suspensions. Therefore, in what follows, we shall focus our attention on this magnitude. Because of the above mentioned difference in demagnetizing effects, we shall compare the apparent yield stress obtained in both geometries at the same internal magnetic fields, $H$. The experimental dependencies of the apparent yield stress on the internal magnetic field are shown in Fig.3a for the MR suspension of particle volume fraction, $\Phi=0.3$. As seen in this figure, in both geometries, the apparent yield stress increases with an increasing magnetic field, which is understood in terms of field-induced enhancement of magnetic interactions, which makes the particle structures more robust and more resistant to shearing forces. At the same internal magnetic fields, the apparent yield stress in the cone-plate geometry (in the magnetic field parallel to the velocity gradient) appears to be somewhat higher than the one in the cylindrical Couette geometry (in the magnetic field longitudinal with the vorticity). This fact seems to be quite intuitive. As already mentioned, in the cone-plate geometry, the field creates particle structures perpendicular to the rheometer walls. These structures hinder significantly the flow and generate substantial viscous dissipation. On the other hand, in the cylindrical Couette geometry, the magnetic field orients the particle structures preferably along the vorticity, so a smaller stress level is expected. However, if the magnetic field is strong enough to induce the particle structures, and if these structures are perfectly aligned with the field, the intensity of the magnetic field in this second case should not influence the stress level of the suspension, and, instead of the Bingham behavior, a Newtonian one is expected with the viscosity defined by the particle volume fraction. Such apparent inconsistency will be explained in the next section. 

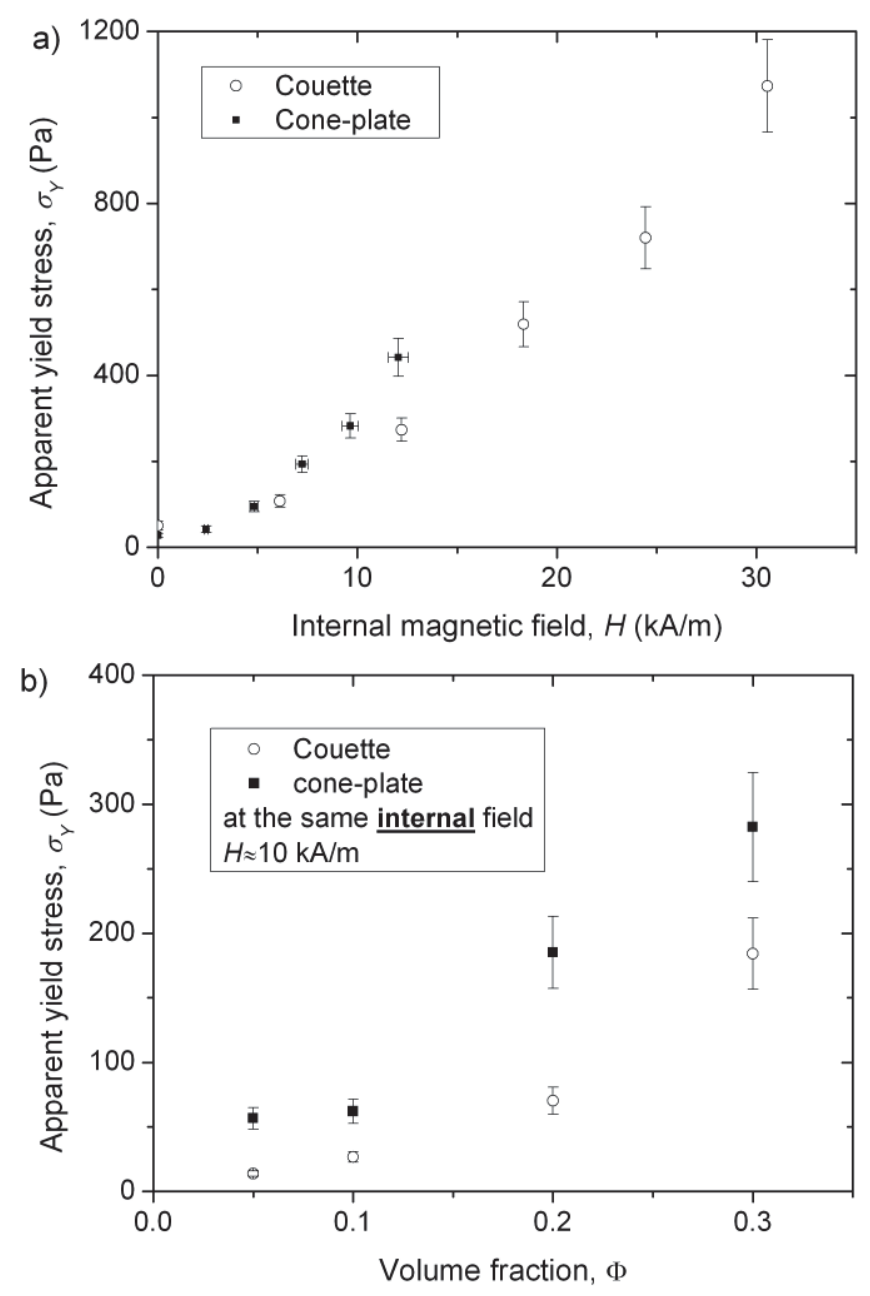

Fig. 3. Comparison of the experimental dependencies of the apparent yield stress on the internal magnetic field (a), and on the particle volume fraction (b), obtained for the cone-plate and the cylindrical Couette geometries. For the results presented in (a), the particle volume fraction is $\Phi=0.3$. For the results presented in (b), the internal magnetic field is adjusted to $H \approx 10 \mathrm{kA} / \mathrm{m}$. The internal magnetic field is calculated in Appendix B as a function of the MR sample shape, its magnetic properties and the external field.

Experimental dependencies of the apparent yield stress on the particle volume fraction, $\Phi$, are shown in Fig. $3 \mathrm{~b}$ for both geometries at the same internal magnetic field, $H \approx 10 \mathrm{kA} / \mathrm{m}$. Obviously, in both cases the MR effect is a growing function of the concentration. Within the concentration range $0.05 \leq \Phi \leq 0.3$, the stress level appears to be larger in cone-plate geometry, as compared to the Couette one, for the reasons explained above. A detailed analysis of the magnetic field and concentration effects on the apparent yield stress in Couette geometry will be studied in details in the next section IV.

\section{Theory and comparison with experiments}

In this section we focus on the appearance of the apparent yield stress when the field is applied in the direction of vorticity. Theoretical explanations for the field-induced apparent yield stress when the field is applied in the direction of the velocity gradient are extensively 
reported in the literature [cf. the review by Bossis et al. (2002b) and the references therein] and will not be treated here.

Qualitatively, we adopt the same explanation for the MR effect in the magnetic field aligned with the vorticity as the one used by Kuzhir et al. (2011) for the magnetic field aligned with the velocity. If, for some reason, the particle aggregates get misaligned with the vorticity, they will generate substantial hydrodynamic stresses proportional to the square of their length-to-diameter ratio, $r_{e}$, a parameter called aspect ratio: $\sigma \propto \eta_{0} \dot{\gamma} r_{e}^{2}$. Once misaligned with the vorticity, tensile hydrodynamic forces start acting on the aggregates, such that their length, or rather aspect ratio, decreases with the shear rate as follows: $r_{e} \propto \dot{\gamma}^{-1 / 2}$ [Shulman et al. (1986), Martin and Anderson (1996), Gómez-Ramírez et al. (2011)]. Therefore, the hydrodynamic part of the aggregate stress appears to be independent of the shear rate and can be considered as the apparent yield stress - the stress associated to the hydrodynamic dissipation on the aggregates rather than to a threshold stress required for breaking the structures and to the onset of the flow.

One of the reasons for the aggregate misalignment from the field and vorticity directions may come from magnetic interactions between aggregates. In the presence of an external field, the aggregates possess an induced magnetic moment and, to a first approximation, can be considered as long dipoles with the magnetic poles located at their extremities. In shear flow, the aggregates initially oriented along the vorticity, move with different velocities because their centers of mass displace along different streamlines. The poles of the neighboring aggregates may attract or repel each other. This may cause the aggregates to change their orientation. Such angular motion should not be confounded with continuous spinning under shear flow. As mentioned in the Introduction, the aggregate flipping is avoided by the magnetic torque exerted on the aggregates by the applied magnetic field. In a relatively concentrated suspension, the aggregates are spaced in an irregular manner, their number is important and their length may vary because of the complicated kinetics of their formation/destruction. This will make inter-aggregate interactions stochastic and lead to random fluctuations of their orientation. In summary, the combination of the stochastic misalignment of the aggregates with their high hydrodynamic resistance and eventual destruction by shearing forces may result in a yielding behavior of the MR fluid under the field aligned with the vorticity. Having proposed a qualitative explanation for the MR effect in the considered geometry, in what follows, we shall try, on this basis, to make a quantitative prediction of the MR fluid apparent yield stress in this geometry.

For this purpose, let us consider a simple shear flow between two infinite planes with the external magnetic field, of an intensity $H_{0}$, oriented parallel to the planes and perpendicular to the flow, cf. Fig.4. The Cartesian reference frame is introduced in such a way that the axis " 1 " is oriented along the fluid velocity, the axis " 2 " - along the velocity gradient and the axis " 3 " - along the vorticity and along the applied magnetic field. Because of the infinite size of the channel in the direction " 3 ", the demagnetizing effects are absent and the magnetic field, $H$, inside the MR fluid is the same as the external field: $H=H_{0}$. Note that such 
estimation of the internal magnetic field remains true for the concentric cylinder geometry used in our experiments. Actually, finite element simulations show only $4 \%$ deviation of the internal field $H$ in the measuring gap from the external one $H_{0}$ (cf. Sec. III-A and Appendix B).

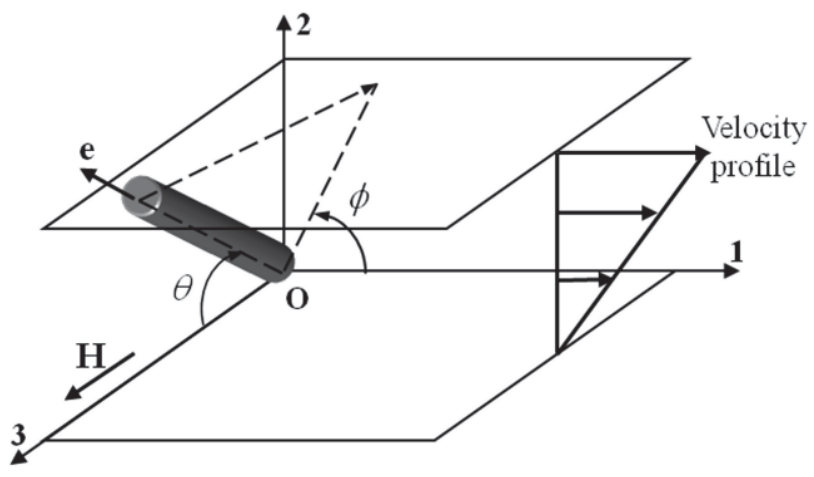

Fig.4. Sketch of the problem geometry

The magnetic field induces the formation of aggregates, of mean length $2 L$ and mean radius $A$, oriented along the applied field. Their stochastic misalignment is supposed to be a Gaussian delta-correlated process and is mimicked by a rotational diffusion process. This diffusion is not caused by Brownian motion of aggregates but is induced by magnetic interactions between neighboring aggregates, resulting in their stochastic angular displacement. According to the random walk principle [van de Ven (1989)], the rotational diffusion process may be seen as a series of stochastic angular "jumps" of the aggregate orientation characterized by a mean square amplitude $\left\langle\Delta \theta^{2}\right\rangle$ and a mean duration $\Delta t$. In the framework of this model, the rotary diffusivity associated to this process is defined as [cf. Eq. (9.7) in Doi and Edwards (1986)]:

$$
D_{r} \sim\left\langle\Delta \theta^{2}\right\rangle / \Delta t=\left\langle\omega^{2}\right\rangle \Delta t
$$

where $\left\langle\omega^{2}\right\rangle=\left\langle\Delta \theta^{2}\right\rangle / \Delta t^{2}$ is the mean square angular velocity of the aggregates. The mean duration of the jumps can be estimated as the inverse of the shear rate, $\Delta t \propto \dot{\gamma}^{-1}$, and the mean square angular velocity through the stochastic torque induced by magnetic interactions with

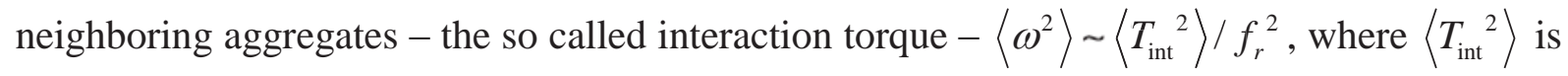
the mean square value of the interaction torque and $f_{r}$ is the rotational friction coefficient of the aggregate. This leads to the following expression for the rotary diffusivity:

$$
D_{r} \propto \frac{\left\langle T_{\mathrm{int}}{ }^{2}\right\rangle}{f_{r}^{2} \dot{\gamma}}
$$

The mean square interaction torque can be estimated using an effective field approach as follows. Consider an aggregate moving in the shear flow and subjected to a magnetic field 
H. The neighboring aggregates moving around our given aggregate will induce a supplementary magnetic field at the location of the given aggregate. This field is expected to be a sum of a permanent component, $\mathbf{H}_{p}$, and a stochastic component $\mathbf{H}_{s t}$. The first one could be attributed to the statistical average interaction between the given aggregate and a surrounding effective medium having a permanent mean magnetization, $\mathbf{M}$. The term $\mathbf{H}_{p}$ may be therefore seen as a local Lorenz field, whose components are equal to $\left(H_{p}\right)_{i}=n_{i k} M_{k}$, with $n_{i k}$ being the tensor of demagnetization factors of the aggregate [Berthier (1993)]. The field $\mathbf{H}_{p}$ contributes to an enhancement of the suspension magnetization due to regular interactions between aggregates and exists even in the absence of fluctuations in their orientation. The second term, $\mathbf{H}_{s t}$, appears due to stochastic magnetic interactions between the aggregates because of irregular spacing between their magnetic poles under shear flow. The orientation of this stochastic field is expected to experience random fluctuations during time, such that $\left\langle\mathbf{H}_{\text {st }}\right\rangle=\mathbf{0}$, while its quadratic mean value is supposed to be proportional to the square of the suspension magnetization: $\left\langle H_{\text {st }}{ }^{2}\right\rangle \sim M^{2}$. The instantaneous interaction torque induced by the stochastic field is therefore given by a vector product of the aggregate magnetic moment and the stochastic field: $\mathbf{T}_{\text {int }}=\mathbf{m} \times \mathbf{H}_{\text {st }}$. The aggregate magnetic moment and the suspension magnetization can be estimated for the case of a perfect alignment of the aggregates with the magnetic field, as follows: $m=\mu_{0} \chi_{a} H V_{a}$ and $M=\left(\Phi / \Phi_{a}\right) \chi_{a} H$ with $\mu_{0}=4 \pi \cdot 10^{-7} \mathrm{H} / \mathrm{m}$ being the magnetic permeability of vacuum; $\chi_{a}$ - the aggregate magnetic susceptibility, $V_{a}=2 \pi A^{2} L$ the aggregate volume, $\Phi$ and $\Phi_{a}$ - the volume fraction of particles in the suspension and the internal volume fraction of the aggregates, so that the ratio $\left(\Phi / \Phi_{a}\right)$ stands for the concentration of aggregates in the suspension. Performing the necessary substitutions and taking into account the expression $f_{r}=8 \pi \eta_{0} L^{3} /(3 \ln \xi)$ for the friction coefficient, we obtain the following expression for the rotary diffusivity:

$$
D_{r}=\alpha\left(\frac{\Phi \mu_{0} \chi_{a}{ }^{2} H^{2}}{\Phi_{a} \eta_{0} \beta}\right)^{2} \frac{1}{\dot{\gamma}}
$$

where $\eta_{0}$ is the viscosity of the suspending liquid, $\beta=4 r_{e}^{2} /(3 \ln \xi)$ is the form-factor describing the hydrodynamic resistance of the aggregates and coming from the slender body theory [Batchelor (1970)]; $\xi$ is the hydrodynamic screening length of the aggregates normalized by their radius $A$; the numerical constant $\alpha$ is introduced as a correction factor called interaction constant, which is taken as a free parameter of the present model. The interaction constant should not be confounded with the interaction parameter, which is often referred to the ratio of the magnetic-to-thermal energy and it is alternatively called the dipolar coupling parameter.

The aggregate aspect ratio $r_{e}$ or rather the form-factor $\beta$, can be found by a balance between tensile hydrodynamic forces and cohesive magnetic forces, as usually done in classical magnetorheology [Shulman et al. (1986), Martin and Anderson (1996)]. For this 
purpose, let us consider an aggregate whose orientation is described by the angles $\theta$ and $\phi$ in the spherical coordinate system, as shown in Fig.4, where $\theta$ is the angle that the aggregate forms with the magnetic field (axis " 3 ") and $\phi$ is the angle between the flow direction (axis "1") and the aggregate projection onto the shear plane "12". Alternatively, the aggregate orientation cay be described by a unit vector, e , oriented along its major axis. The projections of this vector onto the three basis axes read: $e_{1}=\sin \theta \cos \phi, e_{2}=\sin \theta \sin \phi, e_{3}=\cos \theta$. Applied to the geometry considered in the present work, the instantaneous force balance [derived in details in Kuzhir et al. (2011)] reads:

$$
\frac{\pi L^{2}}{\ln \xi} \eta_{0} \dot{\gamma} \sin ^{2} \theta \sin \phi \cos \phi=\frac{3}{2} \pi A^{2} \Phi_{a} f_{m} \cos ^{2} \theta,
$$

where the left-hand part corresponds to the tensile hydrodynamic force and the right-hand part to the magnetic cohesive force; $f_{m}$ is the magnetic force between neighboring particles constituting the aggregates per unit cross-sectional area of the particles. The magnetic force $f_{m}$ and the aggregate magnetic susceptibility $\chi_{a}$, intervening into Eqs. (3) and (4), are calculated as a function of the magnetic field $H$ using finite element simulations, as explained in details in Kuzhir et al. (2011). The simulation results for $f_{m}$ and $\chi_{a}$ have been fitted by the following expressions, valid in the range of the magnetic field intensities, $0 \leq H \leq 30 \mathrm{kA} / \mathrm{m}$ : $f_{m}(H)=\left(6.66 \cdot 10^{4}\left(H / M_{S}\right)^{2}-6.32 \cdot 10^{3}\left(H / M_{S}\right)+168\right) \mu_{0} H^{2}$ and $\chi_{a}(H)=-7.67 \cdot 10^{2}\left(H / M_{S}\right)^{2}-50.9\left(H / M_{S}\right)+9.29$, with $M_{S}=1.36 \cdot 10^{6} \mathrm{~A} / \mathrm{m}$ being the saturation magnetization of the carbonyl iron particles.

During their motion, the aggregates are continuously destroyed by the hydrodynamic forces and reformed by attractive magnetic forces. The average aggregate length is defined by the kinetics of this process rather than by the instantaneous force balance (4). At this stage, we do not need to describe this kinetics in order to understand the mechanism of the MR effect in the case of the magnetic field aligned with the vorticity. A rapid estimate of the aggregate length can be obtained by assuming the precession of an aggregate around the vorticity axis at some precession angle, $\theta$. This aggregate is supposed to be destroyed once it achieves the polar angle, $\phi=\pi / 4$, at which the tensile hydrodynamic force is maximal. Expressing the aggregate aspect ratio $r_{\mathrm{e}}=L / A$ from Eq. (4), as a function of the precession angle $\theta$ and averaging over all possible values of $\theta$, we get the following equation for the form-factor $\beta$ :

$$
\beta \equiv \frac{4 r_{e}^{2}}{3 \ln \xi}=\frac{2 \Phi_{a} f_{m}}{\eta_{0} \dot{\gamma}}\left\langle\cot ^{2} \theta\right\rangle \approx \frac{4 \Phi_{a} f_{m}}{\eta_{0} \dot{\gamma}} \frac{\left\langle e_{3}^{2}\right\rangle}{1-\left\langle e_{3}^{2}\right\rangle},
$$

where the approximation $\left\langle\cot ^{2} \theta\right\rangle \approx\left\langle\cos ^{2} \theta\right\rangle /\left(1-\left\langle\cos ^{2} \theta\right\rangle\right)$ is used, making it possible to express the mean cotangent of the azimuth angle $\theta$ through the mean square component $\left\langle e_{3}^{2}\right\rangle=\left\langle\cos ^{2} \theta\right\rangle$ of the unit vector $\mathbf{e}$. 
The next step is to find the mean orientation of the aggregates under shear and magnetic fields. The aggregate orientation is commonly described by the second and the fourth rang tensors, $\left\langle e_{i} e_{k}\right\rangle$ and $\left\langle e_{i} e_{k} e_{l} e_{m}\right\rangle$ called, respectively, the second and the fourth statistical moments. The equation describing the temporal evolution of the second statistical moment comes from the average of the torque balance and, being applied to the case of long aggregates possessing an induced magnetic moment, reads [Pokrovskiy (1978), Kuzhir et al. (2011)]:

$$
\begin{aligned}
& \frac{d\left\langle e_{i} e_{k}\right\rangle}{d t}=\left[\omega_{i l}\left\langle e_{l} e_{k}\right\rangle-\left\langle e_{i} e_{l}\right\rangle \omega_{l k}\right]+\left[\gamma_{i l}\left\langle e_{l} e_{k}\right\rangle+\left\langle e_{i} e_{l}\right\rangle \gamma_{l k}\right]-2\left\langle e_{i} e_{k} e_{l} e_{m}\right\rangle \gamma_{l m} \\
& +\frac{\mu_{0} H^{2}}{\eta_{0} \beta} \frac{\chi_{a}^{2}\left(1-\Phi / \Phi_{a}\right)}{2+\chi_{a}\left(1-\Phi / \Phi_{a}\right)}\left[h_{i} h_{l}\left\langle e_{l} e_{k}\right\rangle+h_{k} h_{l}\left\langle e_{l} e_{i}\right\rangle-2\left\langle e_{i} e_{k} e_{l} e_{m}\right\rangle h_{l} h_{m}\right]+2 D_{r}\left[\delta_{i k}-3\left\langle e_{i} e_{k}\right\rangle\right]
\end{aligned}
$$

where $t$ is the time; $\gamma_{i k}=(1 / 2) \cdot\left(\partial v_{i} / \partial x_{k}+\partial v_{k} / \partial x_{i}\right)$ and $\omega_{i k}=(1 / 2) \cdot\left(\partial v_{i} / \partial x_{k}-\partial v_{k} / \partial x_{i}\right)$ are the rate-of-strain and vorticity tensors, respectively; $h_{i}$ is the $i$-th component of the unit vector $\mathbf{h}$ oriented along the internal magnetic field $\mathbf{H} ; \delta_{i k}$ is the Kronecker delta. In the present case, we have only two non-zero components of the rate-of-strain and vorticity tensors, $\gamma_{12}=\gamma_{21}=\omega_{12}=-\omega_{21}=\dot{\gamma} / 2$ and one non-zero component of the field unit vector, $h_{3}=1$. The factor $\left[\chi_{a}{ }^{2}\left(1-\Phi / \Phi_{a}\right)\right] /\left[2+\chi_{a}\left(1-\Phi / \Phi_{a}\right)\right]$ appearing in the last equation comes from the magnetic torque exerted on the aggregates by the applied field and has been calculated taking into account the statistical average of the interactions between the given aggregate and the surrounding effective medium (using the above defined Lorenz local field $\mathbf{H}_{p}$ ).

In order to solve equation (6), a closure relation between the second and the fourth statistical moments must be introduced, and we choose the quadratic closure, $\left\langle e_{i} e_{k} e_{l} e_{m}\right\rangle \equiv\left\langle e_{i} e_{k}\right\rangle\left\langle e_{l} e_{m}\right\rangle$, which appears to correctly describe the orientation state of highly aligned suspensions of rod-like particles or molecules [Doi and Edwards (1986)]. Using this relation along with the expressions (3) and (5) for the rotary diffusivity and the aggregate form-factor, respectively, and considering the steady state $\left(d\left\langle e_{i} e_{k}\right\rangle / d t=0\right)$, the equation (6) reduces to the following set of algebraic equations: 


$$
\left\{\begin{array}{l}
\left\langle e_{1} e_{2}\right\rangle-\left\langle e_{1}^{2}\right\rangle\left\langle e_{1} e_{2}\right\rangle-C_{2} \frac{1-\left\langle e_{3}^{2}\right\rangle}{\left\langle e_{3}^{2}\right\rangle}\left\langle e_{1}^{2}\right\rangle\left\langle e_{3}^{2}\right\rangle+C_{1}\left(\frac{1-\left\langle e_{3}^{2}\right\rangle}{\left\langle e_{3}^{2}\right\rangle}\right)^{2}\left(1-3\left\langle e_{1}^{2}\right\rangle\right)=0 \\
-\left\langle e_{2}{ }^{2}\right\rangle\left\langle e_{1} e_{2}\right\rangle-C_{2} \frac{1-\left\langle e_{3}^{2}\right\rangle}{\left\langle e_{3}^{2}\right\rangle}\left\langle e_{2}^{2}\right\rangle\left\langle e_{3}^{2}\right\rangle+C_{1}\left(\frac{1-\left\langle e_{3}^{2}\right\rangle}{\left\langle e_{3}^{2}\right\rangle}\right)^{2}\left(1-3\left\langle e_{2}^{2}\right\rangle\right)=0 \\
-\left\langle e_{3}^{2}\right\rangle\left\langle e_{1} e_{2}\right\rangle+C_{2} \frac{1-\left\langle e_{3}^{2}\right\rangle}{\left\langle e_{3}^{2}\right\rangle}\left(\left\langle e_{3}^{2}\right\rangle-\left\langle e_{3}^{2}\right\rangle^{2}\right)+C_{1}\left(\frac{1-\left\langle e_{3}^{2}\right\rangle}{\left\langle e_{3}^{2}\right\rangle}\right)^{2}\left(1-3\left\langle e_{3}^{2}\right\rangle\right)=0 \\
\left\langle e_{2}^{2}\right\rangle-2\left\langle e_{1} e_{2}\right\rangle^{2}-2 C_{2} \frac{1-\left\langle e_{3}^{2}\right\rangle}{\left\langle e_{3}^{2}\right\rangle}\left\langle e_{1} e_{2}\right\rangle\left\langle e_{3}^{2}\right\rangle-6 C_{1}\left(\frac{1-\left\langle e_{3}^{2}\right\rangle}{\left\langle e_{3}^{2}\right\rangle}\right)^{2}\left\langle e_{1} e_{2}\right\rangle=0
\end{array}\right.
$$

where

$$
C_{1}=\alpha\left[\Phi \mu_{0} \chi_{a}^{2} H^{2} /\left(4 \Phi_{a}^{2} f_{m}\right)\right]^{2}
$$

and

$C_{2}=\mu_{0} \chi_{a}^{2}\left(1-\Phi / \Phi_{a}\right) H^{2} /\left[4 \Phi_{a} f_{m}\left(2+\chi_{a}\left(1-\Phi / \Phi_{a}\right)\right)\right]$ are dimensionless factors independent of the shear rate. As expected, the sum of the left-hand sides of the three first equations gives zero under the condition $\left\langle e_{1}{ }^{2}\right\rangle+\left\langle e_{2}{ }^{2}\right\rangle+\left\langle e_{3}{ }^{2}\right\rangle=1$. The shear rate comes out from equations (6) and (7) because both the magnetic and the diffusion terms (the two last terms at the righthand-side of Eq. (6)) appear to be proportional to the shear rate due to the form-factor $\beta \propto \dot{\gamma}^{-1}$ [cf. Eq. (5)], which makes the rotary diffusivity linear in the shear rate, $D_{r} \propto \beta^{-2} \dot{\gamma}^{-1} \propto \dot{\gamma}$ [cf. Eq. (3)].

The system (7) is solved numerically with respect to the four unknown statistical moments, $\left\langle e_{1}^{2}\right\rangle,\left\langle e_{2}{ }^{2}\right\rangle,\left\langle e_{3}{ }^{2}\right\rangle$ and $\left\langle e_{1} e_{2}\right\rangle$, the components $\left\langle e_{1} e_{3}\right\rangle$ and $\left\langle e_{2} e_{3}\right\rangle$ being zero for symmetry reasons. Analysis shows that the aggregate orientation is strongly influenced by the interaction constant $\alpha$, responsible for the intensity of the rotary diffusion process. Theoretical dependencies of the four second statistical moments on the interaction constant are shown in Fig. 5a in a semi-logarithmic scale for the particle volume fraction $\Phi=0.3$ and the magnetic field, $H=18.3 \mathrm{kA} / \mathrm{m}$. At $\alpha=0$, the diffusion is absent, all the aggregates are perfectly aligned with both the field and the vorticity, which implies $\left\langle e_{3}^{2}\right\rangle=1$ and $\left\langle e_{1}^{2}\right\rangle=\left\langle e_{2}^{2}\right\rangle=\left\langle e_{1} e_{2}\right\rangle=0$. As the interaction constant increases, the diffusion becomes more important, the aggregate orientation exhibits stronger deviation from its equilibrium position, so, the statistical moment $\left\langle e_{3}{ }^{2}\right\rangle$ (mean square cosine of the angle $\theta$ between the aggregates and the field) decreases and the other statistical moments increase. Interestingly, the statistical moment $\left\langle e_{3}{ }^{2}\right\rangle$ passes through a minimum and the moments $\left\langle e_{1} e_{2}\right\rangle$ and $\left\langle e_{1}^{2}\right\rangle$ through a maximum at intermediate values of $\alpha$. At a further increase of the interaction constant, the diffusion becomes very strong and makes the orientation distribution more and more isotropic, such that the three 
diagonal components become equal: $\left\langle e_{1}^{2}\right\rangle=\left\langle e_{2}^{2}\right\rangle=\left\langle e_{3}^{2}\right\rangle=1 / 3$, and the component $\left\langle e_{1} e_{2}\right\rangle$ decreases to zero.
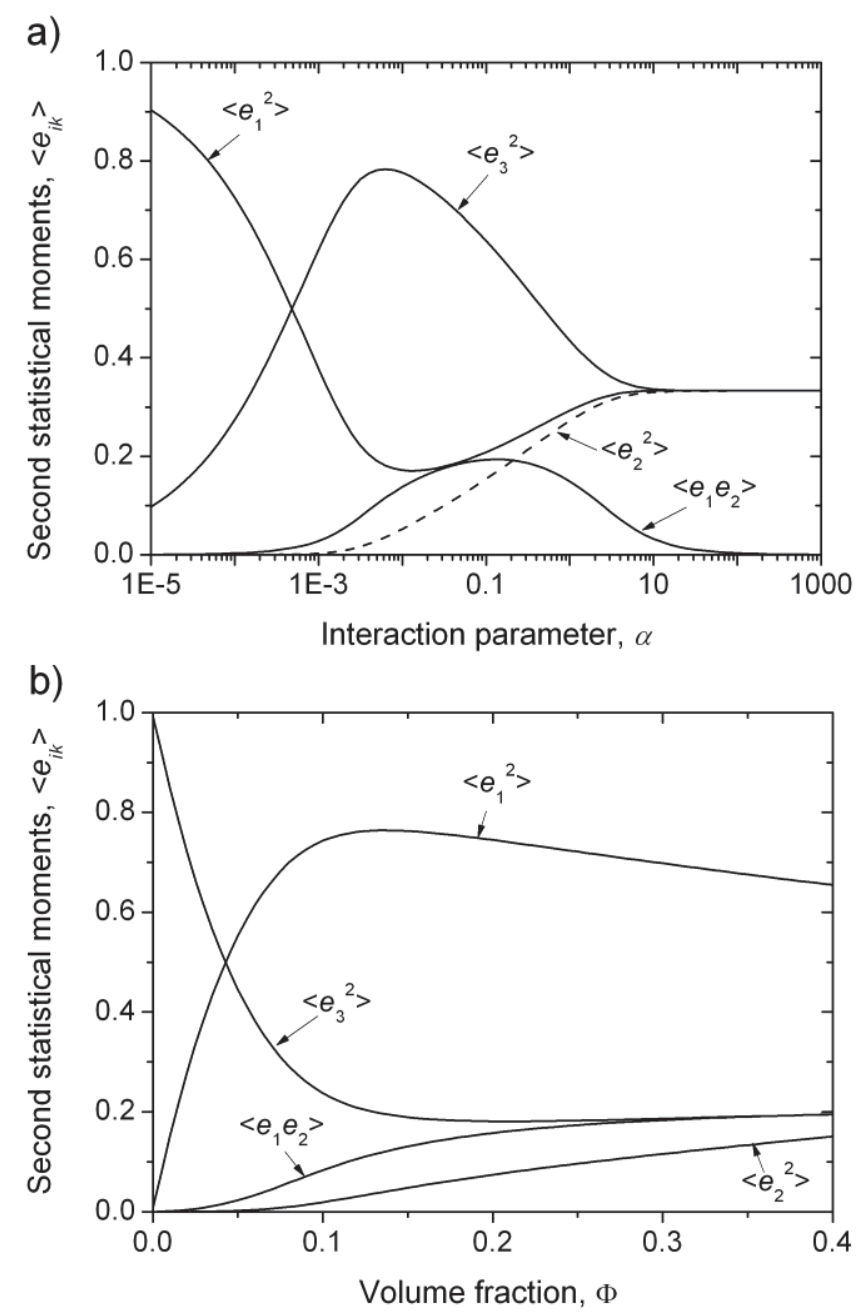

Fig.5. Theoretical dependencies of the second statistical moments on the interaction constant (a) and on the suspension volume fraction (b). In both cases, the magnetic field is parallel to the vorticity and its intensity is equal to $H=18.3 \mathrm{kA} / \mathrm{m}$. In figure (a), the particle volume fraction $\Phi=0.3$; in figure (b), the interaction constant is equal to $\alpha=0.045$.

Another parameter affecting the orientation distribution is the suspension volume fraction, $\Phi$. Theoretical dependencies of the second statistical moments on the volume fraction are presented in Fig. $5 \mathrm{~b}$ for the magnetic field $H=18.3 \mathrm{kA} / \mathrm{m}$ and a fixed value of the interaction constant, $\alpha=0.045$. In the limit of infinite dilution, $\Phi \rightarrow 0$, the aggregates do not feel the presence of each other, and their orientation does not deviate from the equilibrium one - along the vorticity axis - which results in $\left\langle e_{3}^{2}\right\rangle=1$. As the concentration increases, the magnetic interactions between the aggregates increase, the rotary diffusivity grows as $\Phi^{2}$ [cf. Eq. (3)], which leads to an increase of stochastic fluctuations of their orientation. This implies an initial grows of the statistical moments $\left\langle e_{1}^{2}\right\rangle,\left\langle e_{2}^{2}\right\rangle$ and $\left\langle e_{1} e_{2}\right\rangle$ with the concentration and 
a decrease of the moment $\left\langle e_{3}^{2}\right\rangle$. Certainly, at high concentrations, when the orientation state becomes more isotropic, collisions between aggregates may occur. This may change the diffusion process in a way that the orientation distribution will achieve the isotropic state at lower concentrations. We expect, however, that the diffusion process with collisions will keep the same qualitative behavior as the one solely induced by magnetic interactions.

The last stage of our modeling is the calculation of the shear stress. Applied to the case of long aggregates with induced magnetic moments subjected to both shear and magnetic fields, the expression for the stress tensor of a semi-dilute suspension reads [Pokrovskiy (1978), Kuzhir et al. (2011)]:

$$
\begin{aligned}
\sigma_{i k} & =-p \delta_{i k}+2 \eta_{0} \gamma_{i k}+\frac{\Phi}{\Phi_{a}} \eta_{0}\left\{4 \gamma_{i k}+\frac{\beta}{2}\left[\left\langle e_{i} e_{k} e_{l} e_{m}\right\rangle-\frac{1}{3} \delta_{i k}\left\langle e_{l} e_{m}\right\rangle\right] \gamma_{l m}\right\}+ \\
& +\frac{\Phi}{\Phi_{a}} \mu_{0} H^{2} \frac{\chi_{a}^{2}\left(1-\Phi / \Phi_{a}\right)}{2+\chi_{a}\left(1-\Phi / \Phi_{a}\right)}\left[\left\langle e_{i} e_{k} e_{l} e_{m}\right\rangle h_{l} h_{m}-h_{i} h_{l}\left\langle e_{l} e_{k}\right\rangle\right]+\frac{\Phi}{\Phi_{a}} \beta \eta_{0} D_{r}\left[3\left\langle e_{i} e_{k}\right\rangle-\delta_{i k}\right]
\end{aligned}
$$

where $p$ is the pressure in the suspension. Replacing the diffusion constant and the form-factor $\beta$ with appropriate expressions, and using the quadratic closure approximation, equation (8) reduces to the Bingham rheological law for the shear stress $\left(\sigma_{12}\right.$ component of the stress tensor), as follows:

$$
\begin{gathered}
\sigma_{12}=\sigma_{Y}+\eta_{0} \dot{\gamma}\left(1+2 \frac{\Phi}{\Phi_{a}}\right) \\
\sigma_{Y}=2 \Phi f_{m}\left\langle e_{1} e_{2}\right\rangle^{2} \frac{\left\langle e_{3}{ }^{2}\right\rangle}{1-\left\langle e_{3}{ }^{2}\right\rangle}+\frac{\Phi}{\Phi_{a}} \cdot \frac{\chi_{a}{ }^{2}\left(1-\Phi / \Phi_{a}\right)}{2+\chi_{a}\left(1-\Phi / \Phi_{a}\right)} \mu_{0} H^{2}\left\langle e_{1} e_{2}\right\rangle\left\langle e_{3}{ }^{2}\right\rangle+ \\
+\frac{3}{4} \alpha \frac{\Phi^{3}}{\Phi_{a}{ }^{3}} \frac{\left(\mu_{0} \chi_{a}{ }^{2} H^{2}\right)^{2}}{f_{m}} \frac{1-\left\langle e_{3}{ }^{2}\right\rangle}{\left\langle e_{3}{ }^{2}\right\rangle}\left\langle e_{1} e_{2}\right\rangle
\end{gathered}
$$

The apparent yield stress, $\sigma_{Y}$, is the sum of the contributions coming from the hydrodynamic stress, the external torque exerted on the aggregates by the applied magnetic field, and the rotary diffusion of the aggregates, which tends to force the orientation distribution to an isotropic state; these three contributions correspond to the first, the second and the third terms on the right-hand side of Eq. (9b). Replacing the second statistical moments in Eq. (9b) by appropriate numerical values found from Eq. (7), we are able to calculate numerical values of the apparent yield stress as a function of the magnetic field and the particle volume fraction. The theoretical dependency of the three yield stress components on the magnetic field are shown in Fig. 6a for the MR fluid of particle volume fraction $\Phi=0.3$ and for the interaction constant, $\alpha=0.045$. We see that the stress component coming from the rotary diffusion of aggregates seems to be the most important and the stress component coming from the magnetic torque is the least. In figure $6 \mathrm{~b}$, we compare the theoretical and 
experimental field dependencies of the total apparent yield stress for three different particle volume fractions. Our model fits reasonably well the experimental data, and the best fit is obtained with the value $\alpha=0.045$ of the interaction constant. As expected, the apparent yield stress grows monotonically with the magnetic field intensity due to increasing magnetic interactions both between neighboring aggregates and between the particles belonging to the same aggregate.
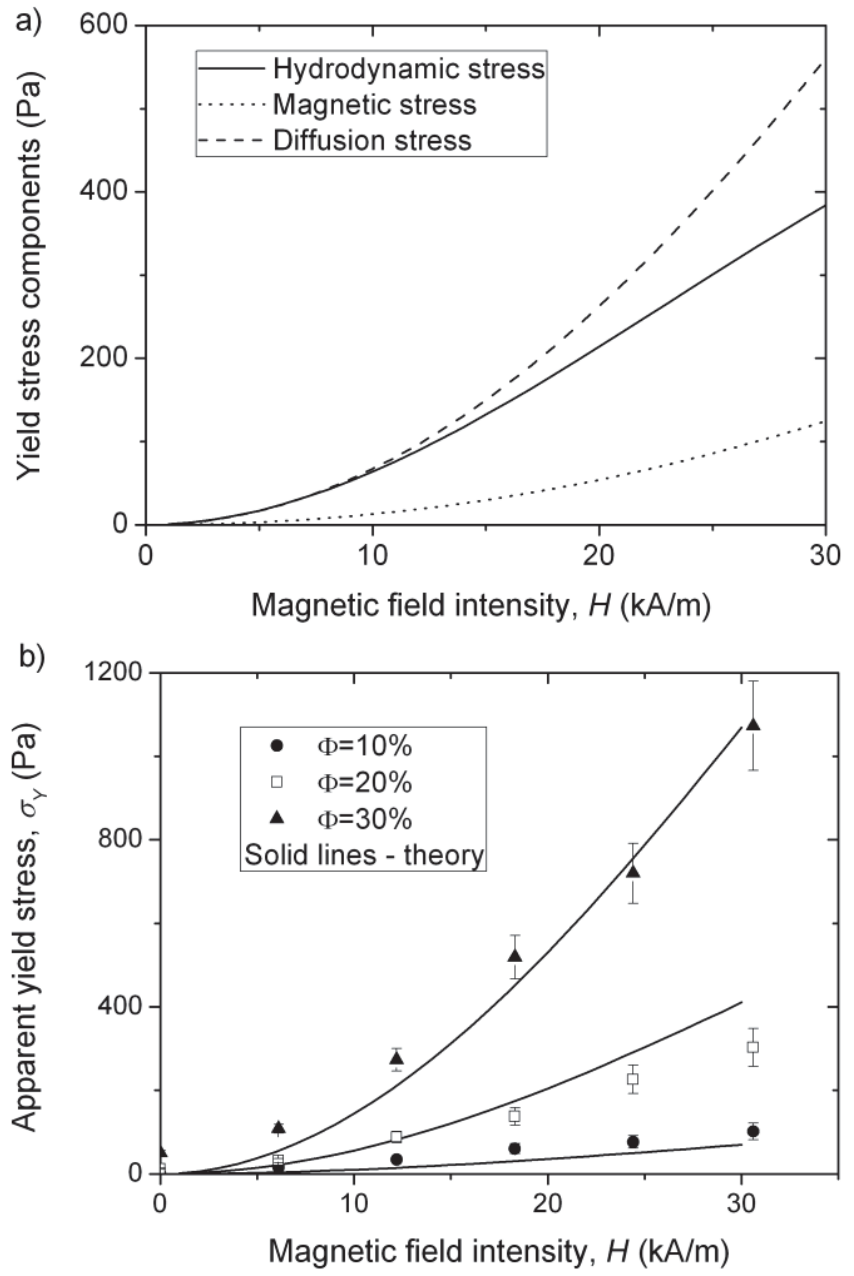

Fig.6. Magnetic field dependencies of the apparent yield stress in the case of the magnetic field aligned with the vorticity. The figure (a) shows three different components of the apparent yield stress calculated with the help of Eq. (9b) at the particle volume fraction $\Phi=0.3$. Figure (b) illustrates a comparison between experiments and theory for the total apparent yield stress at different particle concentrations. In both figures the value $\alpha=0.045$ of the interaction constant is used, which corresponds to the best fit of the theory to the experimental data.

The theoretical and experimental dependencies of the apparent yield stress on the particle volume fraction are presented in Fig. 7 for the magnetic field, $H=18.3 \mathrm{kA} / \mathrm{m}$. The theoretical curve is calculated using the value $\alpha=0.045$ of the adjustable parameter, similar to the one used for the field dependency of the apparent yield stress [cf. Fig. 6b]. Both the experiments and the theory show a nearly quadratic growth of the apparent yield stress with the concentration; the power-law dependency, $\sigma_{Y} \propto \Phi^{2.2}$, is better distinguished in the inset of the Fig.7 where the apparent yield stress is plotted versus $\Phi^{2.2}$. Such a power-law 
concentration dependency can be explained by a combination of two effects as follows. Firstly, in the semi-dilute regime, the stress is roughly proportional to the concentration of aggregates, and thus, to the particle volume fraction. Secondly, with growing concentration, the fluctuations of the aggregate orientation become more important, resulting in an increase of the statistical moment $\left\langle e_{1} e_{2}\right\rangle$ [cf. Fig. 5b]. Since the apparent yield stress is also a growing function of $\left\langle e_{1} e_{2}\right\rangle$ [cf. Eq.9b], both effects lead to a concentration dependency stronger than linear.

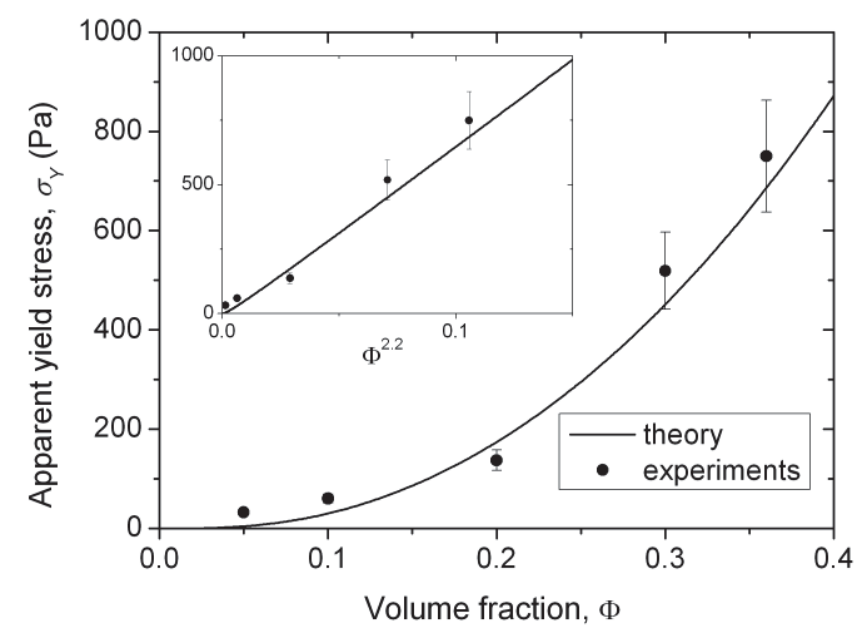

Fig. 7. Experimental and theoretical dependencies of the apparent yield stress on the particle volume fraction in the case of the magnetic field aligned with the vorticity. The magnetic field intensity is equal to $H=18.3 \mathrm{kA} / \mathrm{m}$ and the interaction constant is $\alpha=0.045$

Note that the theoretical results for the concentrated suspensions have been obtained considering a semi-dilute regime and neglecting eventual collisions between aggregates. Such approximations apparently do not induce misleading results and allow us to capture the main physics behind the unexpected appearance of the apparent yield stress in the magnetic field aligned with the vorticity. However, the theory should be improved in the future by considering lubrication contact forces between aggregates and their collisions. Note that collisions between rod-like particles may also result in rotary diffusion, often reported for concentrated suspensions of non-Brownian fiber suspensions [cf. original paper of Folgar and Tucker (1984)]. Both our mechanism and the collision-induced rotary diffusions lead to the same scaling dependencies for the diffusion constant, $D_{r} \propto \dot{\gamma}$. However, the physics is quite different in both cases. In the case of fiber suspensions, the random orientation fluctuations are only dictated by the collision rate between particles proportional to the shear rate, while in our case, it is the interplay between long-range dipolar forces and shear forces, which produces the same scaling.

It is worth mentioning that a similar "rotational diffusion" concept has been previously employed to explain the unexpectedly high MR effect in the magnetic field parallel to the flow through capillaries [Kuzhir et al. (2011)]. We expect that the mechanism of orientation fluctuations is also inherent to the MR response in the magnetic field parallel to the velocity 
gradient (and perpendicular to the walls). In this later case, the dynamic yield stress is conventionally attributed to viscous dissipation on the aggregates inclined with respect to the flow. Conventional models consider a stable steady-state aggregate orientation defined by the equilibrium of the magnetic and hydrodynamic torques. This approach usually leads to relatively small angles between the aggregates and the flow and, consequently, to underestimated values of the apparent yield stress in the perpendicular field [Bossis et al. (2002b)]. In reality, the aggregate orientation is expected to undergo fluctuations because of magnetic interactions and collisions between them. This effect should lead to a more isotropic orientation distribution and to higher stress levels as compared to those predicted by classical theories.

Another important effect, which may influence the rheology at any field orientation, is connected to the reorganization of the internal aggregate structure under flow. The hydrodynamic tensile forces tend to extend the aggregate along their major axis. When the aggregates are progressively misaligned from the direction of the applied field, the tensile forces increase and cause an increase of the internal mechanical stresses. This can induce a progressive rupture of the interparticle contacts and decrease the magnetic cohesive force with the aggregate rotation. A similar effect occurs in the absence of flow, during the elastic deformation of the gap-spanning particle structures as they are extended by the motion of the rheometer plates. In the presence of flow, this effect can modify the force balance (4) and, consequently, the aspect ratio of the aggregates, as well as their orientation distribution. Therefore, further improvement of the theory should include the contribution of local magnetic forces between pairs of particles related to the extension and collisions between aggregates as well as generalization to all orientations of the magnetic field.

\section{Conclusions}

In this work, we have studied the MR fluid rheology in the magnetic field parallel to the fluid vorticity. Experimentally, the MR fluid flow was induced in the Couette coaxial cylinder geometry with the magnetic field parallel to the symmetry axis. The rheological measurements were compared to those obtained in the cone-plate geometry with the magnetic field perpendicular to the lower rheometer plate. Experiments revealed a strong shear-thinning behavior in both geometries with the stress level being just a few dozens of percent smaller in the Couette cylindrical geometry at the same internal magnetic field. This result agrees qualitatively with some preliminary experiments reported by Bossis et al. (2002a). The main goal of the present work is the explanation to the unexpectedly high MR response in the magnetic field parallel to the fluid vorticity.

The key hypothesis is that the particle aggregates are not perfectly aligned with the vorticity, but undergo stochastic fluctuations of their positions and orientations. These fluctuations are induced by magnetic interactions between aggregates. Irregular spacing between the moving aggregates along with their polydispersity impart a stochastic nature to fluctuations. Once misaligned from the vorticity direction, the aggregates generate high hydrodynamic and diffusion stresses, the later being the measure of the stochastic interaction 
torque. Since the aggregate aspect ratio, $r_{e}$, is a decreasing function of the shear rate $\dot{\gamma}$, the stress generated by the aggregates appears to be independent of $\dot{\gamma}$ (in the range of $\dot{\gamma}$ where $r_{e} \gg 1$ ) and it is considered as the suspension apparent (dynamic) yield stress.

Quantitatively, the fluctuations of the aggregate orientation are modeled as a rotary diffusion process with a diffusion constant proportional to the mean square interaction torque. The orientation distribution is found from the equations relating the second and the fourth statistical moments using the quadratic closure approximation. The aggregate mean aspect ratio is found from the balance of the hydrodynamic tensile and magnetic cohesive forces acting on particles constituting the aggregates. The model gives a satisfactory agreement with the experimental field and concentration dependencies of the dynamic yield stress, employing a single adjustable parameter. The rather strong concentration dependency, $\sigma_{Y} \propto \Phi^{2.2}$ revealed by the experiments, is explained by increasing interactions between aggregates leading to stronger misalignments from the vorticity direction with a growing particle concentration.

Further development of the theory will be related to the modeling of the rupture of contacts between particles inside the aggregates due to the extension and collisions between the aggregates subjected to hydrodynamic forces, as well as generalization of the theory to all orientations of the magnetic fields. The practical interest of this study lies in the development of MR smart devices with the magnetic field non-perpendicular to the channel walls.

\section{Acknowledgements}

This work has been supported by Projects P09-FQM-4787 (Junta de Andalucía, Spain), "Factories of the Future" (Grant No. 260073, DynExpert FP7) and PICS 161801 project: "Magnetic nanocomposites for mechanical and biological applications" with Ural Federal University, Russia. In addition, L. Rodríguez-Arco acknowledges financial support by Secretaría de Estado de Educación, Formación Profesional y Universidades (MECD, Spain) through its FPU and Estancias Breves programs.

\section{Appendix A. Magnetic permeability of the aggregated suspension}

Following the work of Gómez-Ramírez et al. (2011), we assume that the applied magnetic field creates cylindrical aggregates of very high aspect ratio $r_{e}>>1$, aligned with the applied magnetic field in the absence of shear. The diagonal components of the magnetic permeability tensor ( $\mu_{\|}$along the aggregate's major axis and $\mu_{\perp}$ along the minor axis) are found using the Maxwell-Garnett mean field theory [Berthier (1993)]:

$$
\begin{aligned}
& \mu_{\|}=1+\chi_{a} \Phi / \Phi_{a}, \\
& \mu_{\perp}=\frac{2+\chi_{a}\left(1+\Phi / \Phi_{a}\right)}{2+\chi_{a}\left(1-\Phi / \Phi_{a}\right)}
\end{aligned}
$$


Recall that $\Phi_{a} \approx \pi / 6$ is the internal volume fraction of the aggregates and the aggregate magnetic susceptibility $\chi_{a}$ is given as a function of the internal magnetic field by the formula cited below Eq. (4).

For calculation of the internal magnetic field in the cylindrical Couette geometry, we shall neglect dispersion of the aggregate orientation with respect to the direction of the applied field. Under such a condition, the axial (along the symmetry axis) and the radial components of the magnetic permeability tensor are simply given by: $\mu_{z z}=\mu_{\|}$and $\mu_{r r}=\mu_{\perp}$.

In the cone-plate geometry, the aggregates are misaligned from the applied field by an angle $\phi$, whose value appears to be independent of the shear rate (in the high-shear regime) and given by [Gómez-Ramírez et al. (2011)]:

$$
\tan ^{2} \phi=\frac{2 f_{m}}{\mu_{0} H^{2}} \frac{2+\chi_{a}\left(1-\Phi / \Phi_{a}\right)}{\chi_{a}{ }^{2}\left(1-\Phi / \Phi_{a}\right)},
$$

where the magnetic force between particles $f_{m}$ per unit particle cross section is defined as a function of the internal field by the formula given below Eq. (4). Note that in the original paper of Gómez-Ramírez et al. (2011), Eq. (A.3) is written in a slightly different form [Eq. (26) in their paper], in which the magnetic force $f_{m}$ is already replaced by a specific expression.

The axial and radial components of the magnetic permeability tensor in the cone-plate geometry are related to the main components $\mu_{\|}$and $\mu_{\perp}$ and to the angle $\phi$ by the following expressions:

$$
\begin{gathered}
\mu_{z z}=\mu_{\|} \cos ^{2} \phi+\mu_{\perp} \sin ^{2} \phi=\frac{\mu_{\|}+\mu_{\perp} \tan ^{2} \phi}{1+\tan ^{2} \phi}, \\
\mu_{r r}=\mu_{\perp},
\end{gathered}
$$

where $\tan ^{2} \phi$ can be directly substituted into Eq. (A.4) from Eq. (A.3).

\section{Appendix B. Distribution of the internal magnetic field}

The magnetic field distribution inside the MR sample is found by numerical solution of Maxwell magnetostatic equations using a finite element method and with the help of FEMM software [Meeker (2009)]. In simulations we use the real geometry of the MR sample and real parameters of the coil applying the external magnetic field $H_{0}$ (magnetic field in the center of the empty coil), as well as the values of the axial and radial components, $\mu_{z z}$ and $\mu_{r r}$, of the suspension magnetic permeability tensor, which have been estimated in Appendix A.

Figure B1a shows the magnetic field distribution in the cylindrical Couette geometry at $H_{0}=30.6 \mathrm{kA} / \mathrm{m}$ (corresponding to an intensity of the electric current of the coil equal to $I=5 \mathrm{~A})$ and at the particle volume fraction $\Phi=0.3$. We remark that the magnetic field is 
relatively homogeneous inside the measuring gap and appears to be rather close to the external field $H_{0}$. This is clearly seen in Fig. B1b, where the field distribution in the measuring gap along the axial direction is compared to the external field. At the same time, we confirm that the magnetic field in the regions below and above the measuring gap is a few times lower that the field inside the gap. Interestingly, the average value of the magnetic field inside the gap seems to be rather insensitive to the magnetic permeability value, $\mu_{z z}=5.4$ (structured suspension) or $\mu_{z z}=2.7$ (unstructured suspension), while the difference between the fields inside the gap and in the MR sample regions above or below the gap is higher for a higher magnetic permeability.
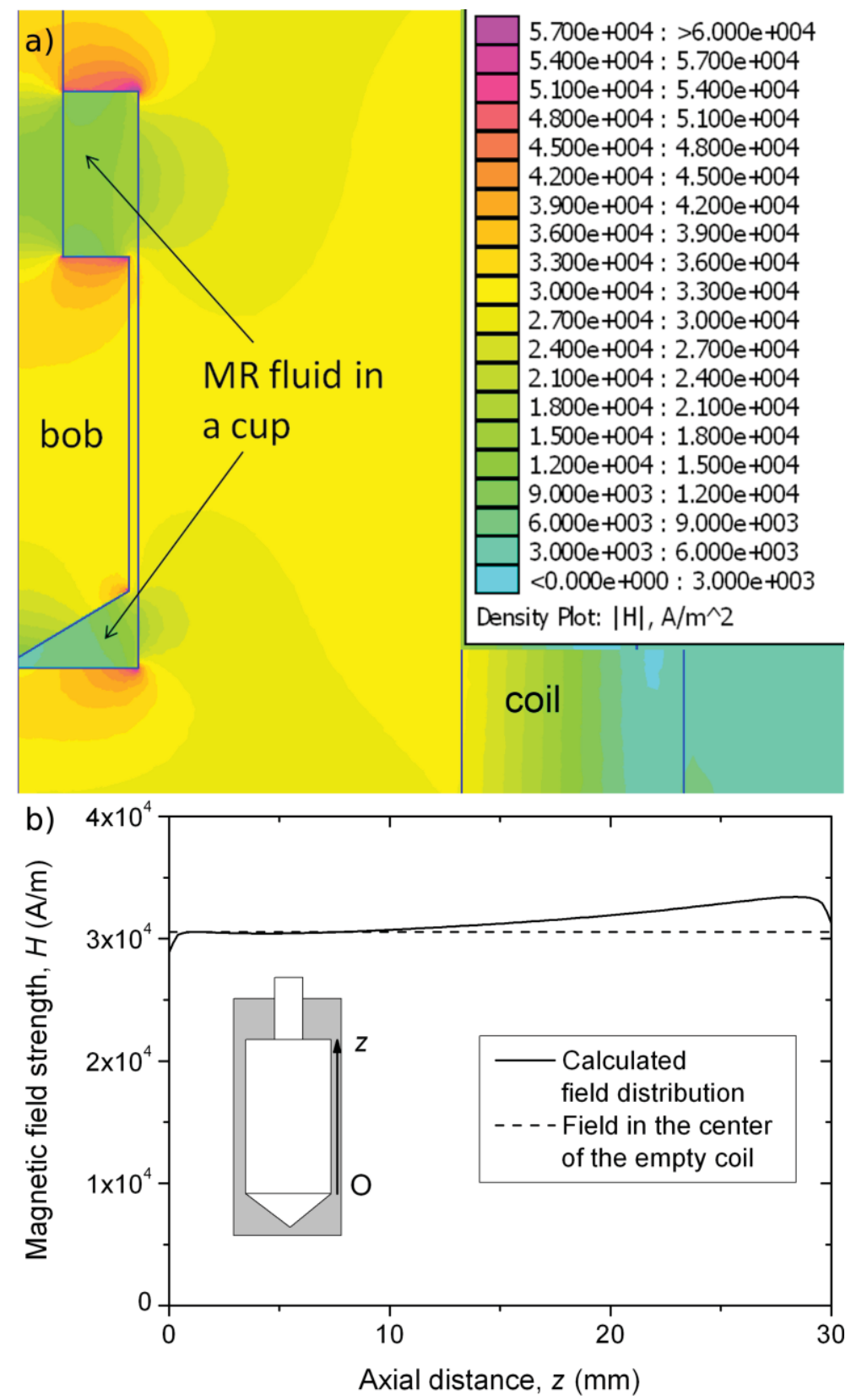

Fig. B1. Magnetic field distribution in the cylindrical Couette geometry at the external field oriented coaxially with the geometry axis of symmetry: 2D distribution (a) and 1D distribution along the vertical axis in the middle between the cylindrical surfaces of the measuring gap (b). The electric current supplied to the coil is $I=5 \mathrm{~A}$; the 
magnetic field in the center of the empty coil is $H_{0}=30.6 \mathrm{kA} / \mathrm{m}$; the particle volume fraction of the MR suspension is $\Phi=0.3$.

Figure B2a shows the magnetic field distribution in the cone-plate geometry at the same values of $H_{0}$ and $\Phi$ as in Fig. B1. The magnetic field also seems to be rather uniform in the measuring gap. As seen in Fig. B1b, its average value is quite close to the estimated one, $H=H_{0} / \mu_{\mathrm{zz}}$.
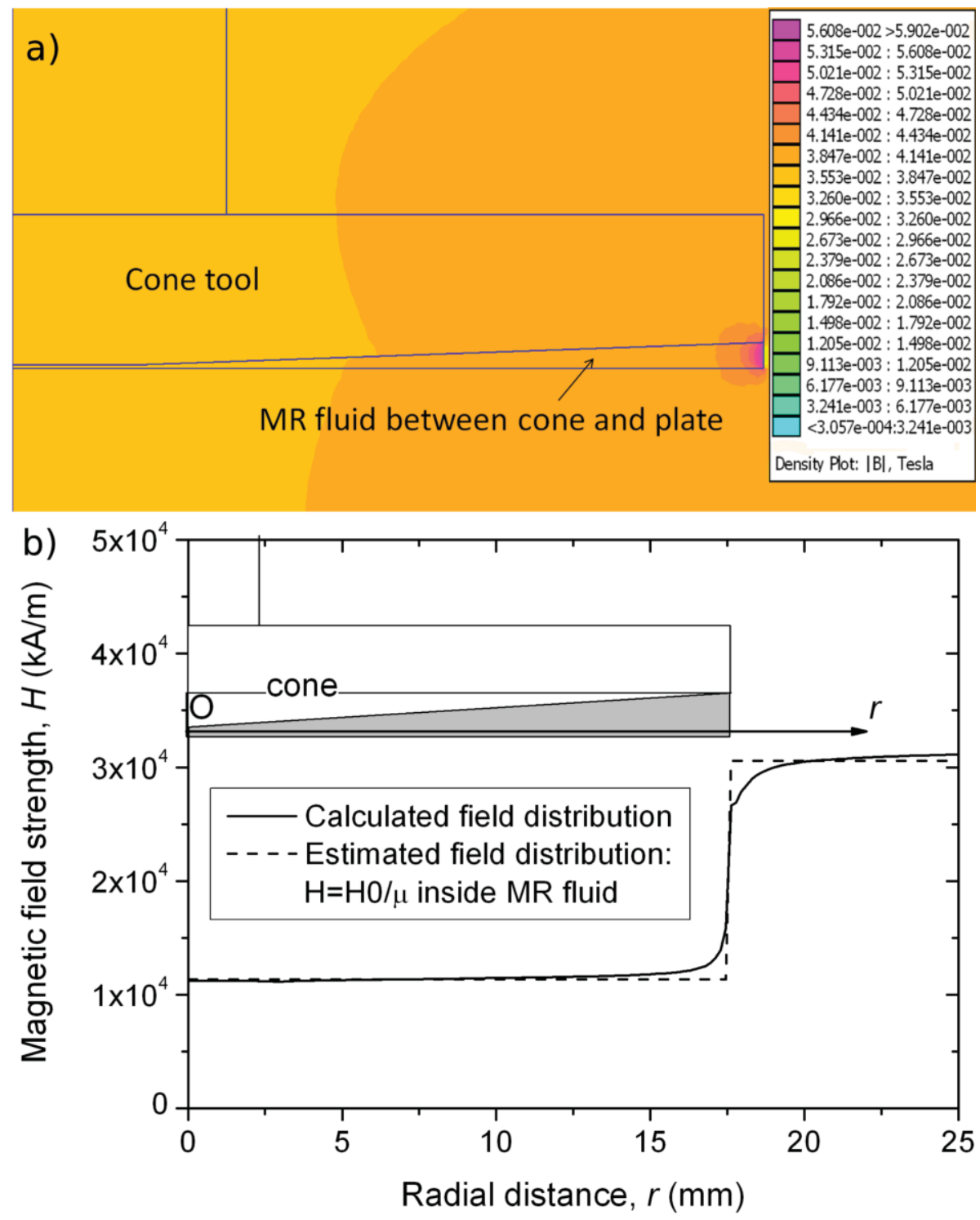

Fig. B2. Magnetic field distribution in the cone-plate geometry with the external field oriented coaxially with the geometry axis of symmetry: 2D distribution (a) and 1D distribution along the radial direction in the middle between the lower surface and the flat part of the upper surface of the measuring gap (b). The electric current supplied to the coil is $I=5 \mathrm{~A}$; the magnetic field in the center of the empty coil is $H_{0}=30.6 \mathrm{kA} / \mathrm{m}$; the particle volume fraction of the MR suspension is $\Phi=0.3$. 


\section{References}

Almog Y. and I. Frankel, "The motion of axisymmetric dipolar particles in homogeneous shear flow", J. Fluid Mech. 289, 243-261 (1995)

Batchelor G.K., "Slender-body theory for particles of arbitrary cross-section in Stokes flow", J. Fluid. Mech. 44, 419-440 (1970)

Berthier, S., Optique des milieux composites, Polytechnica, Paris (1993).

Bossis G., S. Lacis, A. Meunier, and O. Volkova, "Magnetorheological fluids", J.Magn.Magn.Mater. 252, 224-228 (2002-a).

Bossis G., O. Volkova, S. Lacis, and A. Meunier, in "Ferrofluids," Magnetorheology: Fluids, Structures and Rheology, edited by S. Odenbach, Springer, Berlin, (2002-b).

Carlson J.D., D. M. Catanzarite, and K. A. St. Clair, "Commercial magnetorheological fluid devices," Int. J. Mod. Phys. B 10, 2857 (1996).

Chaffey C.E. and S.G. Mason, "Particle behavior in shear and electric fields. III. Rigid spheroids and disks", J. Colloid Sci. 19, 525-548 (1964)

Christensen R.M. Mechanics of composite materials, Krieger Publishing Company, Malabar (1991)

Christensen R.M., «Effective viscous flow properties for fiber suspensions under concentrated conditions », J. Rheol. 37, 103-121 (1993)

de Vicente J., D. J. Klingenberg and R. Hidalgo-Álvarez, "Magnetorheological Fluids: A Review", Soft Matter, 7, 3701-3710 (2011)

de Vicente J., G. Bossis, S. Lacis and M. Guyot, "Permeability Measurements in Cobalt Ferrite and Carbonyl Iron Powders and Suspensions", J. Magn. Magn. Mater., 251, 100-108 (2002)

Doi M. and S.F. Edwards, The theory of polymer dynamics, Oxford University Press, New York, 1986

Folgar, F., and C. L. Tucker, "Orientation behavior of fibers in concentrated suspensions,” J. Reinf. Plast. Compos. 3, 98-119 (1984).

Gavin, H. P., "Annular Poiseuille flow of electrorheological and magnetorheological materials,” J. Rheol. 45, 983-994 (2001).

Genç S. and P.P. Phulé, "Rheological properties of magnetorheological fluids", Smart Mater. Struct. 11, 140-146 (2002).

Ginder J. M., "Behavior of magnetorheological fluids", MRS Bull., 23 26-29 (1998).

Ginder, J. M., L. C. Davis, and L. D. Elie, "Rheology of magnetorheological fluids: Models and measurements," Int. J. Mod. Phys. B 10, 3293-3303 (1996).

Gómez-Ramírez A., P. Kuzhir M.T. López-López, A. Meunier, G. Bossis and J.D.G Durán, "Steady shear flow of magnetic fiber suspensions: Theory and comparison with experiments", J. Rheol. 55, 43-67 (2011).

Kordonski W and S. Gorodkin, "Magnetorheological measurements with consideration for the internal magnetic field in samples", J. Phys: Conf. Series 149, 012064 (2009)

Kordonski W. I. and S. D. Jacobs, "Magnetorheological finishing,” Int. J. Mod. Phys. B 10, 2837 (1996). 
Kordonskii V. I., S. R. Gorodkin, and N. A. Protasevich, "Orientation effects in the flow of a magnetorheological suspension in a magnetic field”, Magnitnaya Gidrodinamika 25, 133-135 (1989); Magnetohydrodynamics 25, 261-264 (1989)

Kuzhir P., C. Magnet, G. Bossis, A. Meunier and V. Bashtovoi, "Rotational diffusion may govern the rheology of magnetic suspensions », J. Rheol. 55, 1297-1318 (2011)

Kuzhir P., G. Bossis, V. Bashtovoi, and O. Volkova, "Effect of the orientation of the magnetic field on the flow of magnetorheological fluid. II. Cylindrical channel", J.Rheol, 47, 1385-1398 (2003).

Laun H.M., C. Kormann and N. Willenbacher, "Rheometry on magnetorheological (MR) fluids. I. Steady shear flow in stationary magnetic fields", Rheol. Acta 35, 417-432 (1996)

López-López M.T., P. Kuzhir, P. Mingalev, and G. Bossis, "Preparation of well dispersed magnetorheological fluids and effect of dispersion on their magnetorheological properties”, Rheol. Acta 47, 787-796 (2008).

Martin J.E., R.A. Anderson, Chain model of electrorheology, J.Chem.Phys 104 48144827 (1996).

Meeker, D. C., Finite Element Method Magnetics, Version 4.2 (Mathematica Build, 15 July 2009), http://www.femm.info.

Ocalan M. and G.H. McKinley, "Rheology and microstructural evolution in pressuredriven flow of magnetorheological fluid with strong particle-wall interactions", J. Intel. Mater. Syst. Struct. 23, 969-978 (2012)

Pokrovskiy V.N., Statistical mechanics of diluted suspensions, Nauka, Moscow 1978.

Shulman Z.P., V.I. Kordonsky, E.A. Zaltsgendler, I.V. Prokhorov, B.M. Khusid and S.A. Demchuk, "Structure, physical properties and dynamics of magnetorheological suspensions”, Int J. Multiphase Flow, 12, 935-955 (1986).

Shulman, Z. P., and W. I. Kordonsky, Magnetorheological effect, Nauka i Tehnika, Minsk, 1982 (in Russian)

Takimoto J., H. Takeda, Y. Masubuchi, and K. Koyama, "Stress Rectification in MR Fluids under Tilted Magnetic Field”, Int. J. Mod. Phys. B, 13, 2028-2035 (1999).

Tao R., and K. Huang, "Reducing blood viscosity with magnetic fields", Phys. Rev. E 84, 011905 (2011)

Ulicny J.C., M.A. Golden, Ch. S. Namuduri, and D. Klingenberg, "Transient response of magnetorheological fluids: Shear flow between concentric cylinders", J. Rheol. 49, 87-104 (2005)

Urreta H., Z. Leicht, A. Sanchez, A. Agirre P. Kuzhir and G Magnac, "Hydrodynamic Bearing Lubricated with Magnetic Fluids”, J. Intell. Mater. Syst. Struct, 21 1491-1499 (2010).

Van de Ven G.M., Colloidal hydrodynamics, Academic Press Limited, London (1989).

Vekas L., D. Bica, I. Potencz, D. Gheorghe, O. Bãlãu and M. Rasa, "Concentration and composition dependence of rheological and magnetorheological properties of some magnetic fluids", Progr. Colloid Polym Sci 117, 104-109 (2001).

Wang X. and F. Gordaninejad, "Study of magnetorheological fluids at high shear stress", Rheol. Acta 45, 899-908 (2006). 


\section{Figure captions}

Fig.1. Cylindrical Couette (bob and cup) geometry with the magnetic field applied parallel to the rheometer axis

Fig.2. Comparison between the MR fluid flow curves measured in the cone-plate and the cylindrical Couette geometries at the same external magnetic field $H_{0}=18.3 \mathrm{kA} / \mathrm{m}$ (a), and at the same internal magnetic field $\mathrm{H} \approx 12 \mathrm{kA} / \mathrm{m}$ (b). For both figures, the particle volume fraction is $\Phi=0.3$

Fig. 3. Comparison of the experimental dependencies of the apparent yield stress on the internal magnetic field (a), and on the particle volume fraction (b), obtained for the cone-plate and the cylindrical Couette geometries. For the results presented in (a), the particle volume fraction is $\Phi=0.3$. For the results presented in (b), the internal magnetic field is adjusted to $H \approx 10 \mathrm{kA} / \mathrm{m}$. The internal magnetic field is calculated in Appendix B as a function of the MR sample shape, its magnetic properties and the external field.

Fig.4. Sketch of the problem geometry

Fig.5. Theoretical dependencies of the second statistical moments on the interaction constant (a) and on the suspension volume fraction (b). In both cases, the magnetic field is parallel to the vorticity and its intensity is equal to $H=18.3 \mathrm{kA} / \mathrm{m}$. In figure (a), the particle volume fraction $\Phi=0.3$; in figure (b), the interaction constant is equal to $\alpha=0.045$.

Fig.6. Magnetic field dependencies of the apparent yield stress in the case of the magnetic field aligned with the vorticity. The figure (a) shows three different components of the apparent yield stress calculated with the help of Eq. (9b) at the particle volume fraction $\Phi=0.3$. Figure (b) illustrates a comparison between experiments and theory for the total apparent yield stress at different particle concentrations. In both figures the value $\alpha=0.045$ of the interaction constant is used, which corresponds to the best fit of the theory to the experimental data.

Fig. 7. Experimental and theoretical dependencies of the apparent yield stress on the particle volume fraction in the case of the magnetic field aligned with the vorticity. The magnetic field intensity is equal to $H=18.3 \mathrm{kA} / \mathrm{m}$ and the interaction constant is $\alpha=0.045$

Fig. B1. Magnetic field distribution in the cylindrical Couette geometry at the external field oriented coaxially with the geometry axis of symmetry: 2D distribution (a) and 1D distribution along the vertical axis in the middle between the cylindrical surfaces of the measuring gap (b). The electric current supplied to the coil is $I=5 \mathrm{~A}$; the magnetic field in the center of the empty coil is $H_{0}=30.6$ $\mathrm{kA} / \mathrm{m}$; the particle volume fraction of the MR suspension is $\Phi=0.3$.

Fig. B2. Magnetic field distribution in the cone-plate geometry with the external field oriented coaxially with the geometry axis of symmetry: 2D distribution (a) and 1D distribution along the radial direction in the middle between the lower surface and the flat part of the upper surface of the measuring gap (b). The electric current supplied to the coil is $I=5 \mathrm{~A}$; the magnetic field in the center of the empty coil is $H_{0}=30.6 \mathrm{kA} / \mathrm{m}$; the particle volume fraction of the MR suspension is $\Phi=0.3$. 